\title{
AN INTEGRATED MODEL FOR THE PRODUCTION OF X-RAY TIME LAGS AND QUIESCENT SPECTRA FROM HOMOGENEOUS AND INHOMOGENEOUS BLACK HOLE ACCRETION CORONAE
}

\author{
John J. Kroon \\ School of Physics, Astronomy, and Computational Sciences, George Mason University, \\ Fairfax, VA 22030-4444, USA; jkroon@gmu.edu \\ Peter A. Becker \\ School of Physics, Astronomy, and Computational Sciences, George Mason University, \\ Fairfax, VA 22030-4444, USA; pbecker@gmu.edu
}

Received __; accepted _

submitted to ApJ 


\begin{abstract}
Many accreting black holes manifest time lags during outbursts, in which the hard Fourier component typically lags behind the soft component. Despite decades of observations of this phenomenon, the underlying physical explanation for the time lags has remained elusive, although there are suggestions that Compton reverberation plays an important role. However, the lack of analytical solutions has hindered the interpretation of the available data. In this paper, we investigate the generation of X-ray time lags in Compton scattering coronae using a new mathematical approach based on analysis of the Fourier-transformed transport equation. By solving this equation, we obtain the Fourier transform of the radiation Green's function, which allows us to calculate the exact dependence of the time lags on the Fourier frequency, for both homogeneous and inhomogeneous coronal clouds. We use the new formalism to explore a variety of injection scenarios, including both monochromatic and broadband (bremsstrahlung) seed photon injection. We show that our model can successfully reproduce both the observed time lags and the time-averaged (quiescent) X-ray spectra for Cyg X-1 and GX 339-04, using a single set of coronal parameters for each source. The time lags are the result of impulsive bremsstrahlung injection occurring near the outer edge of the corona, while the time-averaged spectra are the result of continual distributed injection of soft photons throughout the cloud.
\end{abstract}

Subject headings: X-ray time lags - accretion, accretion disks — black hole physics - black hole binaries: coronae 


\section{INTRODUCTION}

Many accretion-powered X-ray sources display rapid variability, coupled with a time-averaged spectrum consisting of a power law terminating in an exponential cutoff at high energies. The ubiquitous nature of the observations suggests a common mechanism for the spectral formation process, regardless of the type of central object (e.g. black hole, neutron star, AGN, etc.). Over the past few decades, the interpretation of the spectral data using steady-state models has demonstrated that the power-law component is most likely due to the thermal Comptonization of soft seed photons in a hot $\left(\sim 10^{8} \mathrm{~K}\right)$ coronal cloud (Sunyaev \& Titarchuk 1980). While the spectral models yield estimates for the coronal temperature and optical depth, they do not provide much detailed information about the geometry and morphology of the plasma. On the other hand, observations of variability,

characterized by time lags and power spectral densities (PSDs), can supplement the spectral analysis, yielding crucial additional information about the structure of the inner region in the accretion flow, where the most rapid variability is generated.

In particular, the study of X-ray time lags, in which the hard photons associated with a given Fourier component arrive at the detector before or after the soft photons, provides a unique glimpse into the nature of the high-frequency variability in the inner region. Fourier time lags offer an ideal tool for studying rapid variability because, unlike short-timescale spectral snapshots, which become noisy due to the shortage of photons in small time bins, the Fourier technique utilizes all of the data in the entire observational time window, which could extend over hundreds or thousands of seconds. Hence the resulting time lag information usually has much higher significance than can be achieved using conventional spectral analysis. 


\subsection{Fourier Time Lags}

The Fourier method for computing time lags from observational data streams in two energy channels was pioneered by van der Klis et al. (1987), who proposed a novel mathematical technique for extracting time lags by creating a suitable combination of the hard and soft Fourier transforms for a given value of the circular Fourier frequency, $\omega$. The method utilizes the Complex Cross-Spectrum, denoted by $C(\omega)$, defined by

$$
C(\omega) \equiv S^{*}(\omega) H(\omega),
$$

where $S$ and $H$ are the Fourier transforms of the soft and hard channel time series, $s(t)$ and $h(t)$, respectively, and $S^{*}$ denotes the complex conjugate. The Fourier transforms are calculated using

$$
S(\omega)=\int_{-\infty}^{\infty} e^{i \omega t} s(t) d t,
$$

and likewise for the hard channel,

$$
H(\omega)=\int_{-\infty}^{\infty} e^{i \omega t} h(t) d t .
$$

The phase lag between the two data streams is computed by taking the argument of $C(\omega)$, which is the argument angle in the complex plane, and the associated time lag, $\delta t$, is obtained by dividing the phase lag by the Fourier frequency. Hence we have the relations

$$
\delta t=\frac{\arg (C)}{2 \pi \nu_{f}}=\frac{\arg \left(S^{*} H\right)}{2 \pi \nu_{f}},
$$

where the Fourier frequency, $\nu_{f}$, is related to the circular frequency $\omega$ via

$$
\nu_{f}=\frac{\omega}{2 \pi} .
$$

As a simple demonstration of the time lag concept, it is instructive to consider the case where the hard and soft channels, $h(t)$ and $s(t)$, are shifted in time by a precise interval $\Delta t$, so that the two signals are related to each via

$$
h(t)=s(t-\Delta t),
$$


where $\Delta t>0$ would indicate a hard time lag. Next we take the Fourier transform of the hard channel time series to obtain

$$
H(\omega)=\int_{-\infty}^{\infty} e^{i \omega t} h(t) d t=\int_{-\infty}^{\infty} e^{i \omega t} s(t-\Delta t) d t .
$$

Introducing a new time variable, $t^{\prime}=t-\Delta t$ with $d t^{\prime}=d t$, allows us to transform the integral in Equation (7) to obtain

$$
H(\omega)=\int_{-\infty}^{\infty} e^{i \omega\left(t^{\prime}+\Delta t\right)} s\left(t^{\prime}\right) d t^{\prime}=e^{i \omega \Delta t} S(\omega) .
$$

It follows from Equation (11) that the resulting complex cross-spectrum is given by

$$
C(\omega)=S^{*}(\omega) e^{i \omega \Delta t} S(\omega)=e^{i \omega \Delta t}|S(\omega)|^{2},
$$

and hence the resulting time lag is (cf. Equation (44))

$$
\delta t=\frac{\omega \Delta t}{\omega}=\Delta t .
$$

This simple calculation confirms that the time lag computed using the Fourier method gives the correct answer when a perfect delay is introduced between the two channels, as expected. It is also important to note that time lags are only produced during a transient. We can see this by setting the hard and soft signals equal to the constants $h_{0}$ and $s_{0}$, respectively, so that $h(t)=h_{0}$ and $s(t)=s_{0}$. In this case, the resulting Fourier transforms $H$ and $S$ have the same phase, and consequently there is no phase lag or time lag. Hence observations of time lags necessarily imply the presence of variability in the observed signal.

\subsection{X-Ray Time Lag Phenomenology}

The fundamental physical mechanism underlying the X-ray time lag phenomenon has been debated for decades, but it is generally accepted that the time lags reflect the time-dependent scattering of a population of seed photons that are impulsively injected 
into an extended corona of hot electrons (e.g., van der Klis et al. 1987; Miyamoto et al. 1988). This initial population of photons gain energy as they Comptonize in the cloud, and the hard time lags are a natural consequence of the extra time that the hard photons spend in the cloud gaining energy via electron scattering before escaping. In contrast with the time lags, the time-averaged (quiescent) spectra are thought to be created as a result of the Compton scattering of continually injected seed photons. The time-dependent upscattering of soft input photons is discussed in detail by Payne (1980) and Sunyaev \& Titarchuk (1980), who present fundamental formulas for the resulting X-ray spectrum. Since that time, many detailed models have been proposed, most of which focus on a single aspect of radiative transfer, usually by making assumptions about the physical conditions in the disk/corona system regarding the electron temperature, the input photon spectrum, and the size and optical depth of the scattering corona.

The Fourier time lags observed from accreting black-hole sources generally decrease with increasing Fourier frequency, $\nu_{f}$. In the case of Cyg X-1, for example, the time lags decrease from $\sim 0.1-10^{-3}$ sec as $\nu_{f}$ increases from $\sim 0.1 \mathrm{~Hz}-10^{2} \mathrm{~Hz}$. Early attempts to interpret this data using simple Compton scattering models resulted in very large, hot scattering clouds, which required very efficient heating at large distances $\left(\sim 10^{5-6} G M / c^{2}\right)$ from the central mass (Poutanen \& Fabian 1999, Hua et al. 1999, hereafter HKC). Furthermore, the observed dependence of the time lags on the Fourier frequency was difficult to explain using a homogeneous Compton scattering model. For example, van der Klis et al. (1987) and Miyamoto et al. (1988) found that a homogeneous corona combined with monochromatic soft photon injection resulted in time lags that are independent of the Fourier frequency, $\nu_{f}$, in contradiction to the observations. This led Miyamoto et al. (1988) to conclude, somewhat prematurely, that thermal Comptonization could not be producing the lags. However, in the next decade, HKC and Nowak et al. (1999) developed more robust Compton simulations that successfully reproduced the observed time lags, although 
the large coronal radii $\sim 10^{4.5-5.5} G M / c^{2}$ continued to raise concerns regarding energy conservation and heating.

HKC computed the time lags and the time-averaged spectra for a variety of electron number density profiles, based on the injection of low-temperature blackbody seed photons at the center of the coronal cloud. They employed a two-region structure, comprising a central homogeneous zone, connected to a homogeneous or inhomogeneous outer region that extends out to several light-seconds from the central mass. In the inhomogeneous case, the electron number density, $n_{e}(r)$, in the outer region varied as $n_{e}(r) \propto r^{-1}$ or $n_{e}(r) \propto r^{-3 / 2}$. In the HKC model, the injection spectrum and the injection location were both held constant, and a zero-flux boundary condition was adopted at the center of the cloud. HKC found that only the model with $n_{e}(r) \propto r^{-1}$ in the outer region was able to successfully reproduce the observed dependence of the time lags on the Fourier frequency. On the other hand, in the homogeneous case, HKC confirmed the Miyamoto et al. (1988) result that the time lags are independent of the Fourier frequency, in contradiction to the observational data. This result was also verified later by Kroon \& Becker (2014, hereafter $\mathrm{KB})$ for the case of monochromatic photon injection into a homogenous corona.

\subsection{Dependence on Injection Model}

Despite the progress made by HKC and other authors, no successful first-principles theoretical model for the production of the observed X-ray time lags has yet emerged. In the absence of such a model, one is completely dependent on Monte Carlo simulations, which are somewhat inconvenient since the resulting time lags are not analytically connected with the parameters describing the scattering cloud. Monte Carlo simulations are also noisy at high Fourier frequency, which is the main region of interest in many applications, although this can be dealt with by adding more test particles. Compared with an analytical 
calculation, the utilization of Monte Carlo simulations makes it more challenging to explore different injection scenarios, such as the variation of the injection location and the seed photon spectrum.

The situation changed recently with the work of KB, who presented a detailed analytical solution to the problem of time-dependent thermal Comptonization in spherical, homogeneous scattering clouds. By obtaining the fundamental photon Green's function solution to the problem, they were able to explore a wide variety of injection scenarios, leading to a better understanding of the relationship between the observed time lags and the underlying physical parameters. KB verified the Miyamoto result, namely that monochromatic injection in a homogeneous cloud produces time lags that are independent of Fourier period. The magnitude of this (constant) lag depends primarily on the radius of the cloud, $R$, its optical thickness, $\tau_{*}$, and the electron temperature, $T_{e}$. Following HKC, they employed a zero-net flux boundary condition at the center of the corona (essentially a mirror condition), so that injection could occur at any radius inside the cloud. The photon transport at the outer edge of the cloud was treated using a free-streaming boundary condition in order to properly account for photon escape. KB demonstrated that the injection radius and the shape of the injected photon spectrum play a crucial role in determining the dependence of the resulting time lags on the Fourier frequency. In particular, they established for the first time that the reprocessing of a broadband injection spectrum (e.g., thermal bremsstrahlung) can successfully reproduce most of the time lag data for Cyg X-1 and other sources.

In the study presented here, we expand on the work of KB to obtain the radiation Green's function for inhomogeneous scattering clouds. We also present a more detailed derivation of the homogeneous Green's function discussed by KB. The analytical solutions for the Fourier transform of the time-dependent Green's function in the homogeneous and 
inhomogeneous cases are then used to treat localized bremsstrahlung injection via integral convolution, as an alternative to the essentially monochromatic injection scenario studied by HKC. In addition to modeling the transient time lags as a result of impulsive soft photon injection, we also compute the time-independent X-ray spectrum radiated form the surface of the cloud as a result of continual soft photon injection. We show that acceptable fits to both the time-lag data and the X-ray spectral data can be obtained using a single set of cloud parameters (temperature, density, cloud radius) via application of our integrated model.

The remainder of the paper is organized as follows. In Section 2 we introduce the time-dependent and steady-state transport equations in spherical geometry, and we map out the general solution methods to be applied in the subsequent sections. In Section 3 we obtain the solution for the Fourier transform of the time-dependent photon Green's function and also the solution for the time-averaged Green's function in a homogeneous corona. In Section 4, we repeat the same steps for the case of an inhomogeneous corona with electron number density profile $n_{e}(r) \propto 1 / r$. We discuss the reprocessing of thermal bremsstrahlung radiation in Section 5, and we apply the integrated model to Cyg X-1 and GX 339-04 in Section 6. Our main conclusions are reviewed and further discussed in Section 7.

\section{Fundamental Equations}

Our focus here is on understanding how time-dependent Compton scattering affects a population of seed photons as they propagate through a spherical corona of hot electrons overlying a geometrically thin, standard accretion disk. This problem was first explored using an exact mathematical approach by KB, who studied the radiative transfer occurring in a homogeneous corona. We provide further details of that work here, and we also extend the model to treat inhomogeneous spherical scattering clouds. 


\subsection{Time-Dependent Transport Equation}

The time-dependent transport equation describing the diffusion and Comptonization of an instantaneous flash of $N_{0}$ monochromatic seed photons injected with energy $\epsilon_{0}$ at radius

$r_{0}$ and at time $t_{0}$ as they propagate through a spherical scattering corona is given by (e.g., Becker 2003),

$$
\begin{aligned}
\frac{\partial f_{\mathrm{G}}}{\partial t}=\frac{1}{r^{2}} \frac{\partial}{\partial r}\left[\kappa(r) r^{2} \frac{\partial f_{\mathrm{G}}}{\partial r}\right]+\frac{n_{e}(r) \sigma_{\mathrm{T}} c}{m_{e} c^{2}} \frac{1}{\epsilon^{2}} \frac{\partial}{\partial \epsilon}\left[\epsilon ^ { 4 } \left(f_{\mathrm{G}}\right.\right. & \left.\left.+k T_{e} \frac{\partial f_{\mathrm{G}}}{\partial \epsilon}\right)\right] \\
& +\frac{N_{0} \delta\left(t-t_{0}\right) \delta\left(r-r_{0}\right) \delta\left(\epsilon-\epsilon_{0}\right)}{4 \pi r_{0}^{2} \epsilon_{0}^{2}}
\end{aligned}
$$

where $m_{e}, n_{e}, T_{e}, k, \sigma_{\mathrm{T}}, c$, and $\kappa$ denote the electron mass, the electron number density, the electron temperature, Boltzmann's constant, the Thomson cross section, the speed of light, and the spatial diffusion coefficient, respectively, and $f_{\mathrm{G}}(\epsilon, r, t)$ is the radiation Green's function, describing the distribution of photons inside the cloud. The first term on the right-hand side of Equation (111) represents the spatial diffusion of photons through the corona, and the second term describes the redistribution in energy due to Compton scattering. The Green's function is related to the photon number density, $n_{r}$, via

$$
n_{r}(r, t)=\int_{0}^{\infty} \epsilon^{2} f_{\mathrm{G}}(\epsilon, r, t) d \epsilon,
$$

and the spatial diffusion coefficient $\kappa(r)$ is related to the electron number density $n_{e}(r)$ and the scattering mean free path $\ell(r)$ via

$$
\kappa(r)=\frac{c}{3 n_{e}(r) \sigma_{\mathrm{T}}}=\frac{c \ell(r)}{3} .
$$

Klein-Nishina corrections are important when the incident photon energy in the electron's rest frame approaches $\sim 500 \mathrm{keV}$. In our model, the electrons are essentially non-relativistic, with temperature $T_{e} \sim 4-7 \times 10^{8} \mathrm{~K}$, and therefore the $0.1-10 \mathrm{keV}$ photons of interest here will not be boosted into the Klein-Nishina energy range in the typical electron's rest frame. We will therefore treat the electron scattering process using the Thomson cross section 
throughout this study. However, we revisit this issue is Section 7.1 where we compare our results with previous studies that utilized the full Klein-Nishina cross section to treat the electron scattering.

\subsection{Density Variation}

In many cases of interest, the electron number density $n_{e}(r)$ has a power-law dependence on the radius $r$, which can be written as

$$
n_{e}(r)=n_{*}\left(\frac{r}{R}\right)^{-\alpha}
$$

where $R$ is the outer radius of the cloud, $\alpha$ is a constant, and $n_{*} \equiv n_{e}(R)$ is the number density at the outer edge of the cloud. The two cases we focus on here are

$$
\alpha= \begin{cases}0, & \text { homogeneous } \\ 1, & \text { inhomogeneous }\end{cases}
$$

The homogeneous case was treated by Miyamoto (1988) and the inhomogeneous case by HKC. By combining Equations (13) and (14), we can rewrite the electron number density and the spatial diffusion coefficient as

$$
n_{e}(r)=\frac{1}{\sigma_{\mathrm{T}} \ell_{*}}\left(\frac{r}{R}\right)^{-\alpha}, \quad \kappa(r)=\frac{c \ell_{*}}{3}\left(\frac{r}{R}\right)^{\alpha}
$$

where

$$
\ell_{*} \equiv \ell(R)=\frac{1}{n_{e}(R) \sigma_{\mathrm{T}}}
$$

denotes the scattering mean free path at the outer edge of the corona. Substituting Equations (16) into Equation (11) yields

$$
\begin{aligned}
\frac{\partial f_{\mathrm{G}}}{\partial t}=\frac{c \ell_{*}}{3 r^{2}} \frac{\partial}{\partial r}\left[\left(\frac{r}{R}\right)^{\alpha} r^{2} \frac{\partial f_{\mathrm{G}}}{\partial r}\right]+\frac{1}{\ell_{*} m_{e} c}\left(\frac{r}{R}\right)^{-\alpha} \frac{1}{\epsilon^{2}} \frac{\partial}{\partial \epsilon}\left[\epsilon^{4}\left(f_{\mathrm{G}}+k T_{e} \frac{\partial f_{\mathrm{G}}}{\partial \epsilon}\right)\right] & +\frac{N_{0} \delta\left(t-t_{0}\right) \delta\left(r-r_{0}\right) \delta\left(\epsilon-\epsilon_{0}\right)}{4 \pi r_{0}^{2} \epsilon_{0}^{2}}
\end{aligned}
$$


The electron temperature $T_{e}$ is determined by a balance between gravitational heating and Compton cooling, and one typically finds that $T_{e}$ does not vary significantly in the region where most of the X-rays are produced (You et al. 2012; Schnittman et al. 2013). We therefore assume that the cloud is isothermal with $T_{e}=$ constant. In this case, it is convenient to rewrite the transport equation in terms of the dimensionless energy

$$
x \equiv \frac{\epsilon}{k T_{e}} .
$$

We also introduce the dimensionless radius $z$, time $p$, and temperature $\Theta$, defined, respectively, by

$$
z \equiv \frac{r}{R}, \quad p \equiv \frac{c t}{\ell_{*}}, \quad \Theta \equiv \frac{k T_{e}}{m_{e} c^{2}} .
$$

The various functions involved in the derivation can be written in terms of either the dimensional energy and radius, $(\epsilon, r)$, or the corresponding dimensionless variables $(x, z)$, and therefore we will use these two notations interchangeably throughout the remainder of the paper. Incorporating Equations (19) and (20) into the transport equation (18) yields, after some algebra, $\frac{\partial f_{\mathrm{G}}}{\partial p}=\frac{1}{3 \eta^{2} z^{2}} \frac{\partial}{\partial z}\left(z^{2+\alpha} \frac{\partial f_{\mathrm{G}}}{\partial z}\right)+\frac{\Theta}{z^{\alpha} x^{2}} \frac{\partial}{\partial x}\left[x^{4}\left(f_{\mathrm{G}}+\frac{\partial f_{\mathrm{G}}}{\partial x}\right)\right]+\frac{N_{0} \delta\left(x-x_{0}\right) \delta\left(p-p_{0}\right) \delta\left(z-z_{0}\right)}{4 \pi z_{0}^{2} R^{3} x_{0}^{2} \Theta^{3}\left(m_{e} c^{2}\right)^{3}}$

where we have introduced the dimensionless "scattering parameter,"

$$
\eta \equiv \frac{R}{\ell_{*}}=n_{e}(R) \sigma_{\mathrm{T}} R
$$

Equation (21) is the fundamental partial differential equation that we will use to treat time-dependent scattering in a homogeneous spherical corona with $\alpha=0$ in Section 3, and time-dependent scattering in an inhomogeneous spherical corona with $\alpha=1$ in Section 4 . 


\subsection{Optical Depth}

The scattering optical depth $\tau$ measured from the inner edge of the coronal cloud at radius $r=r_{\text {in }}$ out to some arbitrary local radius $r$ is computed using

$$
\tau(r)=\int_{r_{\text {in }}}^{r} n_{e}\left(r^{\prime}\right) \sigma_{\mathrm{T}} d r^{\prime}=\int_{r_{\text {in }}}^{r} \frac{d r^{\prime}}{\ell\left(r^{\prime}\right)},
$$

where the variation of the mean-free path is given by (see Equations (13) and (14))

$$
\ell(r)=\ell_{*}\left(\frac{r}{R}\right)^{\alpha} .
$$

Combining relations, and transforming the variable of integration from $r$ to $z=r / R$, we obtain

$$
\tau(z)=\eta \int_{z_{\mathrm{in}}}^{z} \frac{d z^{\prime}}{z^{\prime \alpha}}
$$

where

$$
z_{\text {in }} \equiv \frac{r_{\text {in }}}{R}
$$

denotes the dimensionless inner radius of the cloud.

There are three cases of interest here,

$$
\tau(z)= \begin{cases}\eta\left(z^{1-\alpha}-z_{\text {in }}^{1-\alpha}\right) /(1-\alpha), & \alpha \neq 1, \\ \eta\left(z-z_{\text {in }}\right), & \alpha=0, \\ \eta \ln \left(z / z_{\text {in }}\right), & \alpha=1 .\end{cases}
$$

The overall optical thickness of the scattering cloud, denoted by $\tau_{*}$, as measured from the inner radius $r=r_{\text {in }}\left(z=z_{\text {in }}\right)$ to the outer radius $r=R(z=1)$, is therefore given by

$$
\tau_{*}= \begin{cases}\eta\left(1-z_{\text {in }}^{1-\alpha}\right) /(1-\alpha), & \alpha \neq 1, \\ \eta\left(1-z_{\text {in }}\right), & \alpha=0, \\ \eta \ln \left(1 / z_{\text {in }}\right), & \alpha=1 .\end{cases}
$$




\subsection{Steady-State Transport Equation}

The time-averaged (quiescent) X-ray spectra produced in accretion flows around black holes are generally interpreted as the result of the thermal Comptonization of soft seed photons continually injected into a hot electron corona from a cool underlying disk (see e.g. Sunyaev \& Titarchuk 1980 for a review). In our interpretation, the associated X-ray time lags are the result of the time-dependent Comptonization of seed photons impulsively injected during a brief transient. Our goal in this paper is to develop an integrated model that accounts for the formation of both the time-averaged spectrum and the time lags using a single set of cloud parameters (temperature, density, radius). In our calculation of the time-averaged spectrum, we assume that $\dot{N}_{0}$ seed photons with energy $\epsilon_{0}$ are injected per unit time into the hot corona between the inner cloud radius $r_{\text {in }}$ and the outer cloud radius $r=R$ with a rate that is proportional to the local electron number density $n_{e}(r)$. The radial variation of the number density depends on whether the cloud is homogeneous, with $n_{e}=$ constant, or inhomogeneous, with $n_{e}(r) \propto r^{-1}$.

In this scenario, the fundamental time-independent transport equation can be written as

$$
\frac{\partial f_{\mathrm{G}}^{\mathrm{S}}}{\partial t}=0=\frac{1}{r^{2}} \frac{\partial}{\partial r}\left[\kappa(r) r^{2} \frac{\partial f_{\mathrm{G}}^{\mathrm{S}}}{\partial r}\right]+\frac{n_{e}(r) \sigma_{\mathrm{T}} c}{m_{e} c^{2}} \frac{1}{\epsilon^{2}} \frac{\partial}{\partial \epsilon}\left[\epsilon^{4}\left(f_{\mathrm{G}}^{\mathrm{S}}+k T_{e} \frac{\partial f_{\mathrm{G}}^{\mathrm{S}}}{\partial \epsilon}\right)\right]+\frac{\dot{N}_{0} \delta\left(\epsilon-\epsilon_{0}\right) n_{e}(r)}{\epsilon_{0}^{2} N_{e}},
$$

where $f_{\mathrm{G}}^{\mathrm{S}}(\epsilon, r)$ denotes the steady-state (quiescent) photon Green's function, and

$$
N_{e}=\int_{r_{\text {in }}}^{R} 4 \pi r^{2} n_{e}(r) d r
$$

represents the total number of electrons in the region $r_{\text {in }} \leq r \leq R$. Substituting for $n_{e}(r)$ and $\kappa(r)$ in Equation (29) using Equations (16) yields

$0=\frac{c \ell_{*}}{3 r^{2}} \frac{\partial}{\partial r}\left[\left(\frac{r}{R}\right)^{\alpha} r^{2} \frac{\partial f_{\mathrm{G}}^{\mathrm{S}}}{\partial r}\right]+\frac{1}{\ell_{*} m_{e} c}\left(\frac{r}{R}\right)^{-\alpha} \frac{1}{\epsilon^{2}} \frac{\partial}{\partial \epsilon}\left[\epsilon^{4}\left(f_{\mathrm{G}}^{\mathrm{S}}+k T_{e} \frac{\partial f_{\mathrm{G}}^{\mathrm{S}}}{\partial \epsilon}\right)\right]+\frac{\dot{N}_{0} \delta\left(\epsilon-\epsilon_{0}\right)(r / R)^{-\alpha}}{\sigma_{\mathrm{T}} \ell_{*} \epsilon_{0}^{2} N_{e}}$. 
This expression can be rewritten in terms of the dimensionless parameters $x, z, \Theta$, and $\eta$ to obtain

$$
0=\frac{1}{3 \eta^{2} z^{2-\alpha}} \frac{\partial}{\partial z}\left(z^{2+\alpha} \frac{\partial f_{\mathrm{G}}^{\mathrm{S}}}{\partial z}\right)+\frac{\Theta}{x^{2}} \frac{\partial}{\partial x}\left[x^{4}\left(f_{\mathrm{G}}^{\mathrm{S}}+\frac{\partial f_{\mathrm{G}}^{\mathrm{S}}}{\partial x}\right)\right]+\frac{\dot{N}_{0} \delta\left(x-x_{0}\right)(3-\alpha)}{4 \pi R^{2} \eta c \Theta^{3}\left(m_{e} c\right)^{3} x_{0}^{2}\left(1-z_{\mathrm{in}}^{3-\alpha}\right)},
$$

where we have also substituted for $N_{e}$ using

$$
N_{e}=\frac{4 \pi R^{3}}{\sigma_{\mathrm{T}} \ell_{*}} \frac{1-z_{\mathrm{in}}^{3-\alpha}}{3-\alpha}
$$

which follows from Equations (16) and (30). We assume here that $\alpha=0$ or $\alpha=1$.

The derivative $\partial f_{\mathrm{G}}^{\mathrm{S}} / \partial x$ exhibits a step-function discontinuity at the injection energy, $x=x_{0}$, due to the appearance of the function $\delta\left(x-x_{0}\right)$ in Equation (32). By integrating Equation (32) with respect to $x$ over a small region surrounding the injection energy, we conclude that the derivative jump is given by

$$
\left.\lim _{\delta \rightarrow 0}\left[\frac{d f_{\mathrm{G}}^{\mathrm{S}}}{d x}\right]\right|_{x_{0}-\delta} ^{x_{0}+\delta}=-\frac{\dot{N}_{0}(3-\alpha)}{4 \pi R^{2} \eta c \Theta^{4}\left(m_{e} c\right)^{3} x_{0}^{4}\left(1-z_{\mathrm{in}}^{3-\alpha}\right)} .
$$

We will utilize Equations (32) and (34) in Sections 3 and 4 when we compute the timeaveraged X-ray spectra produced via electron scattering in homogeneous and inhomogeneous scattering coronae, respectively.

\subsection{Fourier Transformation}

In principle, all of the detailed spectral variability due to time-dependent Comptonization in the scattering corona can be computed by solving the fundamental transport equation (21) for a given initial photon energy/space distribution (Becker 2003). However, complete information about the variability of the spectrum is not required, or even desired, if the goal it to compare the theoretically predicted time lags $\delta t$ with the observational data. Computation of the predicted time lags using Equation (44) requires as input the 
Fourier transforms of the soft and hard data streams. It is therefore convenient to analyze the time-dependent transport Equation (21) directly in the Fourier domain, rather than in the time domain. Hence one of our goals is to derive the exact solution for the Fourier transform, $F_{\mathrm{G}}$, of the time-dependent radiation Green's function, $f_{\mathrm{G}}$. We define the Fourier transform pair, $\left(f_{\mathrm{G}}, F_{\mathrm{G}}\right)$, using

$$
\begin{gathered}
F_{\mathrm{G}}(x, z, \tilde{\omega}) \equiv \int_{-\infty}^{\infty} e^{i \tilde{\omega} p} f_{\mathrm{G}}(x, z, p) d p \\
f_{\mathrm{G}}(x, z, p) \equiv \frac{1}{2 \pi} \int_{-\infty}^{\infty} e^{-i \tilde{\omega} p} F_{\mathrm{G}}(x, z, \tilde{\omega}) d \tilde{\omega}
\end{gathered}
$$

where the dimensionless Fourier frequency is defined by

$$
\tilde{\omega}=\omega\left(\frac{\ell_{*}}{c}\right)=\omega t_{*} .
$$

Here, $t_{*}=\ell_{*} / c$ is the "scattering time," which equals the mean-free time at the outer edge of the corona, at radius $r=R$.

We can obtain an ordinary differential equation satisfied by the Fourier transform, $F_{\mathrm{G}}$, by operating on Equation (21) with $\int_{-\infty}^{\infty} e^{i \tilde{\omega} p} d p$, to obtain

$$
\begin{aligned}
-i \tilde{\omega} z^{\alpha} F_{\mathrm{G}}=\frac{1}{3 \eta^{2} z^{2-\alpha}} \frac{\partial}{\partial z}\left(z^{2+\alpha} \frac{\partial F_{\mathrm{G}}}{\partial z}\right)+\frac{\Theta}{x^{2}} \frac{\partial}{\partial x}\left[x ^ { 4 } \left(F_{\mathrm{G}}\right.\right. & \left.\left.+\frac{\partial F_{\mathrm{G}}}{\partial x}\right)\right] \\
& +\frac{N_{0} \delta\left(x-x_{0}\right) \delta\left(z-z_{0}\right) e^{i \tilde{\omega} p_{0}}}{4 \pi x_{0}^{2} z_{0}^{2} z^{-\alpha} \Theta^{3}\left(m_{e} c^{2}\right)^{3} R^{3}}
\end{aligned}
$$

where $i^{2}=-1$. Further progress can be made by noting that Equation (38) is separable in the energy and spatial coordinates $(x, z)$. The technical details depend on the value of $\alpha$, which determines the spatial variation of the electron number density $n_{e}(r)$. We therefore treat the homogeneous and inhomogeneous cases separately in Sections 3 and 4, respectively.

Due to the function $\delta\left(x-x_{0}\right)$ appearing in the source term in Equation (38), the energy derivative $\partial F_{\mathrm{G}} / \partial x$ displays a jump at the injection energy $x=x_{0}$, with a magnitude 
determined by integrating Equation (38) with respect to $x$ in a small region around the injection energy. The result obtained is

$$
\left.\lim _{\delta \rightarrow 0}\left[\frac{d F_{\mathrm{G}}}{d x}\right]\right|_{x_{0}-\delta} ^{x_{0}+\delta}=-\frac{N_{0} \delta\left(z-z_{0}\right) e^{i \tilde{\omega} p_{0}}}{4 \pi x_{0}^{4} z_{0}^{2} z^{-\alpha} \Theta^{4}\left(m_{e} c^{2}\right)^{3} R^{3}} .
$$

This expression will be used later in the computation of the expansion coefficients for the Fourier transform of the radiation Green's function resulting from time-dependent Comptonization in Sections 3.2 and 4.2.

\subsection{Boundary Conditions}

In order to obtain solutions for $f_{\mathrm{G}}^{\mathrm{S}}(\epsilon, r)$ and $F_{\mathrm{G}}(\epsilon, r, \tilde{\omega})$, we must impose suitable spatial boundary conditions at the inner edge of the cloud, $r=r_{\mathrm{in}}$, and at the outer edge, $r=R$, which correspond to the dimensionless radii $z=z_{\text {in }}$ and $z=1$, respectively. The boundary conditions we discuss below are stated in terms of the fundamental time-dependent photon Green's function, $f_{\mathrm{G}}(\epsilon, r, t)$, but they also apply to the time-averaged spectrum $f_{\mathrm{G}}^{\mathrm{S}}(\epsilon, r)$. Furthermore, we can show via Fourier transformation that the same boundary conditions also apply to the Fourier transform $F_{\mathrm{G}}(\epsilon, r, \tilde{\omega})$. Note that we can write the time-averaged X-ray spectrum $f_{\mathrm{G}}^{\mathrm{S}}$ and the Fourier transform $F_{\mathrm{G}}$ as functions of either the dimensional energy and radius, $(\epsilon, r)$, or in terms of the dimensionless variables $(x, z)$, and therefore we will use the appropriate set of variables depending on the context.

In the Monte Carlo simulations performed by HKC, the time lags result from the reprocessing of blackbody seed photons impulsively injected at the center of the Comptonizing corona. In order to avoid unphysical sources or sinks of radiation at the center of the cloud, $r=0$, they employed a zero-flux "mirror" inner boundary condition, which can be expressed as

$$
\lim _{r \rightarrow 0}-4 \pi r^{2} \kappa(r) \frac{\partial f_{\mathrm{G}}(\epsilon, r, t)}{\partial r}=0
$$


This condition simply reflects the fact that no photons are created or destroyed at the center of the cloud after the initial flash. Following HKC, we will employ the mirror boundary condition at the center of the corona $(r=0)$ in our calculations involving a homogeneous cloud.

The scattering corona has a finite extent, and therefore we must impose a free-streaming boundary condition at the outer surface $(r=R)$. Hence the distribution function $f_{\mathrm{G}}$ must satisfy the outer boundary condition

$$
-\left.\kappa(r) \frac{\partial f_{\mathrm{G}}(\epsilon, r, t)}{\partial r}\right|_{r=R}=\left.c f_{\mathrm{G}}(\epsilon, r, t)\right|_{r=R},
$$

which implies that the diffusion flux at the surface is equivalent to the outward propagation of radiation at the speed of light.

When the electron distribution is inhomogeneous $\left(n_{e}(r) \propto r^{-1}\right)$, the mirror condition cannot be applied at the center of the cloud due to the divergence of the electron number density $n_{e}(r)$ as $r \rightarrow 0$. In this case, we must truncate the scattering corona at a non-zero inner radius, $r=r_{\text {in }}$, where we impose a free-streaming boundary condition. Physically, the inner edge of the cloud may correspond to the edge of a centrifugal funnel, or the cusp of a thermal condensation feature (Meyer \& Meyer-Hofmeister 2007). The inner free-streaming boundary condition can be written as

$$
-\left.\kappa(r) \frac{\partial f_{\mathrm{G}}(\epsilon, r, t)}{\partial r}\right|_{r=r_{\mathrm{in}}}=-\left.c f_{\mathrm{G}}(\epsilon, r, t)\right|_{r=r_{\mathrm{in}}}
$$

which is only applied in the inhomogeneous case. All of the boundary conditions considered here are satisfied by the fundamental time-dependent photon Green's function $f_{\mathrm{G}}(\epsilon, r, t)$, and also by the time-averaged spectrum $f_{\mathrm{G}}^{\mathrm{S}}(\epsilon, r)$, and the Fourier transform $F_{\mathrm{G}}(\epsilon, r, \tilde{\omega})$. We will apply these results in Sections 3 and 4 where we consider homogeneous and inhomogeneous cloud configurations, respectively. 


\section{Homogeneous Model}

The simplest electron number density distribution of interest here is $n_{e}=$ constant ( $\alpha=0)$, which was first studied by Miyamoto et al. (1988). In this case we apply the mirror inner boundary condition at the center of the cloud, and hence we set $z_{\text {in }}=0$. We consider the homogeneous case in detail in this section, and obtain the exact solutions for the Fourier transform of the time-dependent photon Green's function, $F_{\mathrm{G}}(\epsilon, r, \tilde{\omega})$, and also

for the associated time-averaged radiation spectrum, $f_{\mathrm{G}}^{\mathrm{S}}(\epsilon, r)$. These results were originally presented by KB in an abbreviated form. Note that KB utilized the scattering optical depth $\tau$ measured from the center of the cloud as the fundamental spatial variable, whereas we use the dimensionless radius $z$. However, the two quantities are simply related via Equations (27) and (28), which yield, for $\alpha=0$ and $z_{\text {in }}=0$,

$$
\tau(z)=\eta z, \quad \tau_{*}=\eta
$$

where $\tau_{*}$ is the optical thickness measured from the center of the cloud to the outer edge at $z=1$.

\subsection{Quiescent Spectrum for $\alpha=0$}

In the homogeneous case $(\alpha=0)$, the time-independent transport equation (32) representing the thermal Comptonization of seed photons continually injected throughout the scattering corona can be simplified by substituting the separation functions

$$
f_{\lambda}=K(\lambda, x) Y(\lambda, z)
$$

which yields, for $x \neq x_{0}$,

$$
\frac{-1}{Y \eta^{2} z^{2}} \frac{d}{d z}\left(z^{2} \frac{d Y}{d z}\right)=\frac{3 \Theta}{K x^{2}} \frac{d}{d x}\left[x^{4}\left(K+\frac{d K}{d x}\right)\right]=\lambda,
$$


where $\lambda$ is the separation constant. The corresponding ordinary differential equations satisfied by the spatial and energy functions $Y$ and $K$ are, respectively,

$$
\begin{gathered}
\frac{1}{z^{2}} \frac{d}{d z}\left(z^{2} \frac{d Y}{d z}\right)+\lambda \eta^{2} Y=0 \\
\frac{1}{x^{2}} \frac{d}{d x}\left[x^{4}\left(K+\frac{d K}{d x}\right)\right]-\frac{\lambda}{3 \Theta} K=0,
\end{gathered}
$$

which has been considered previously by such authors as Payne (1980), Shapiro, Lightman, and Eardley (1976), Sunyaev \& Titarchuk (1980), etc.

The fundamental solution for the energy function $K$ is given by (see Becker 2003)

$$
K(\lambda, x)=\left(x x_{0}\right)^{-2} e^{-\left(x+x_{0}\right) / 2} M_{2, \sigma}\left(x_{\min }\right) W_{2, \sigma}\left(x_{\max }\right)
$$

where $M_{2, \sigma}$ and $W_{2, \sigma}$ are Whittaker functions,

$$
x_{\max } \equiv \max \left(x, x_{0}\right), \quad x_{\min } \equiv \min \left(x, x_{0}\right)
$$

and

$$
\sigma \equiv \sqrt{\frac{9}{4}+\frac{\lambda}{3 \Theta}} .
$$

The specific form in Equation (48) represents the solution satisfying appropriate boundary conditions at high and low energies, and it is also continuous at the injection energy, $x=x_{0}$, as required.

In the homogeneous configuration under consideration here, the spatial function $Y$ must satisfy the inner "mirror" boundary condition at the origin (cf. Equation (40)), which can be written in terms of $z$ as

$$
\lim _{z \rightarrow 0} z^{2} \frac{d Y(\lambda, z)}{d z}=0
$$

The fundamental solution for $Y$ satisfying this condition is given by

$$
Y(\lambda, z)=\frac{\sin (\eta z \sqrt{\lambda})}{\eta z} .
$$


By virtue of Equation (41), the spatial function $Y$ must also satisfy the outer free-streaming boundary condition, written in terms of the $z$ coordinate as

$$
\lim _{z \rightarrow 1}\left[\frac{1}{3 \eta} \frac{d Y(\lambda, z)}{d z}+Y(\lambda, z)\right]=0 .
$$

Substituting the form for $Y$ given by Equation (52) into Equation (53) yields a transcendental equation for the eigenvalues $\lambda_{n}$ that can be solved using a numerical root-finding procedure. The resulting eigenvalues $\lambda_{n}$ are all real and positive, and the corresponding values of $\sigma$ are computed by setting $\lambda=\lambda_{n}$ in Equation (50). The associated eigenfunctions, $Y_{n}$ and $K_{n}$, are defined by

$$
Y_{n}(z) \equiv Y\left(\lambda_{n}, z\right), \quad K_{n}(x) \equiv K\left(\lambda_{n}, x\right)
$$

According to the Sturm-Liouville theorem, the eigenfunctions $Y_{n}$ form an orthogonal basis with respect to the weight function $z^{2}$, so that (see Appendix A)

$$
\int_{0}^{1} z^{2} Y_{n}(z) Y_{m}(z) d z=0, \quad n \neq m .
$$

The related quadratic normalization integrals, $\mathscr{I}_{n}$, are defined by

$$
\mathscr{I}_{n} \equiv \eta^{3} \int_{0}^{1} z^{2} Y_{n}^{2}(z) d z=\frac{\eta}{2}-\frac{\sin \left(2 \eta \sqrt{\lambda_{n}}\right)}{4 \sqrt{\lambda_{n}}}
$$

where the final result follows from Equation (52).

Based on the orthogonality of the $Y_{n}$ functions, we can express the time-averaged photon Green's function using the expansion

$$
f_{\mathrm{G}}^{\mathrm{S}}\left(x, x_{0}, z\right)=\sum_{n=0}^{\infty} b_{n} K_{n}(x) Y_{n}(z),
$$

where the expansion coefficients $b_{n}$ are computed using the derivative jump condition in Equation (34). In the case of interest here, we set $\alpha=0$ and $z_{\text {in }}=0$ to obtain

$$
\left.\lim _{\delta \rightarrow 0}\left[\frac{d f_{\mathrm{G}}^{\mathrm{S}}}{d x}\right]\right|_{x_{0}-\delta} ^{x_{0}+\delta}=-\frac{3 \dot{N}_{0}}{4 \pi R^{2} \eta c \Theta^{4}\left(m_{e} c\right)^{3} x_{0}^{4}} .
$$


Substituting the series expansion for the steady-state Green's function (Equation (57)) into Equation (58) yields

$$
\lim _{\delta \rightarrow 0} \sum_{n=0}^{\infty} b_{n} Y_{n}(z)\left[K_{n}^{\prime}\left(x_{0}+\delta\right)-K_{n}^{\prime}\left(x_{0}-\delta\right)\right]=-\frac{3 \dot{N}_{0}}{4 \pi R^{2} \eta c \Theta^{4}\left(m_{e} c\right)^{3} x_{0}^{4}} .
$$

We can make further progress by eliminating $K$ using Equation (48) to obtain, after some algebra,

$$
\sum_{n=0}^{\infty} b_{n} Y_{n}(z) \mathscr{W}_{2, \sigma}\left(x_{0}\right)=-\frac{3 \dot{N}_{0} e^{x_{0}}}{4 \pi R^{2} \eta c \Theta^{4}\left(m_{e} c\right)^{3}},
$$

where we have defined the Wronskian of the Whittaker functions using

$$
\mathscr{W}_{2, \sigma}\left(x_{0}\right) \equiv M_{2, \sigma}\left(x_{0}\right) W_{2, \sigma}^{\prime}\left(x_{0}\right)-W_{2, \sigma}\left(x_{0}\right) M_{2, \sigma}^{\prime}\left(x_{0}\right)
$$

The Wronskian can be evaluated analytically to obtain (Abramowitz \& Stegun 1970)

$$
\mathscr{W}_{2, \sigma}\left(x_{0}\right)=-\frac{\Gamma(1+2 \sigma)}{\Gamma(\sigma-3 / 2)}
$$

Combining Equations (60) and (62), we obtain

$$
\sum_{n=0}^{\infty} b_{n} Y_{n}(z) \frac{\Gamma(1+2 \sigma)}{\Gamma(\sigma-3 / 2)}=\frac{3 \dot{N}_{0} e^{x_{0}}}{4 \pi R^{2} \eta c \Theta^{4}\left(m_{e} c^{2}\right)^{3}} .
$$

Next we exploit the orthogonality of the $Y_{n}$ functions with respect to the weight function $z^{2}$ by applying the operator $\int_{0}^{1} \eta^{3} z^{2} Y_{m}(z) d z$ to both sides of Equation (63). According to Equation (55), all of the terms on the left-hand side vanish except the term with $m=n$. The result obtained for the expansion coefficient $b_{n}$ is therefore

$$
b_{n}=\frac{3 \dot{N}_{0} e^{x_{0}} \Gamma(\sigma-3 / 2) \mathscr{P}_{n}}{4 \pi R^{2} \eta c \Theta^{4}\left(m_{e} c^{2}\right)^{3} \Gamma(1+2 \sigma) \mathscr{I}_{n}},
$$

where the integrals $\mathscr{I}_{n}$ are computed using Equation (156) and the integrals $\mathscr{P}_{n}$ are defined by

$$
\mathscr{P}_{n} \equiv \int_{0}^{1} \eta^{3} z^{2} Y_{n}(z) d z=\frac{3 \eta \sin \left(\eta \sqrt{\lambda_{n}}\right)}{\lambda_{n}}
$$


and the final result follows from application of Equation (53).

Combining Equations (57) and (64) yields the exact analytical solution for the time-independent photon Green's function evaluated at dimensionless energy $x$ and dimensionless radius $z$ resulting from the continual injection of seed photons throughout the cloud. We obtain

$$
f_{\mathrm{G}}^{\mathrm{S}}\left(x, x_{0}, z\right)=\frac{9 \dot{N}_{0} e^{x_{0}}}{4 \pi R^{2} c \Theta^{4}\left(m_{e} c^{2}\right)^{3}} \sum_{n=0}^{\infty} \frac{\Gamma(\sigma-3 / 2) \sin \left(\eta \sqrt{\lambda_{n}}\right)}{\lambda_{n} \Gamma(1+2 \sigma) \mathscr{I}_{n}} K_{n}(x) Y_{n}(z)
$$

where $\sigma$ is computed using Equation (150), and $Y_{n}$ and $K_{n}$ are defined in Equation (54). This is the same result as Equation (27) from KB, once we make the identifications $\tau_{*}=\eta$ and $G_{n}(\tau)=Y_{n}(z)$, which arise due to the change in the spatial variable from the dimensionless radius $z$ used here, to the scattering optical depth $\tau=\eta z$ used by KB. The time-averaged X-ray spectrum computed using Equation (66) is compared with the observational data for Cyg X-1 and GX 339-04 in Section 6.1. In Section 6.1.2, we use asymptotic analysis to derive a power-law approximation to the exact radiation distribution

given by Equation (66), and we show that the resulting approximate X-ray spectrum agrees closely with that obtained using the exact solution.

\subsection{Fourier Transform for $\alpha=0$}

In the homogeneous case $(\alpha=0)$, we can substitute for the Fourier transform $F_{\mathrm{G}}$ in Equation (38) using the separation functions

$$
F_{\lambda} \equiv H(\lambda, x) Y(\lambda, z)
$$

to obtain, for $x \neq x_{0}$,

$$
-\frac{1}{Y} \frac{1}{\eta^{2} z^{2}} \frac{d}{d z}\left(z^{2} \frac{d Y}{d z}\right)=\frac{3 \Theta}{H x^{2}} \frac{d}{d x}\left[x^{4}\left(H+\frac{d H}{d x}\right)\right]+3 i \tilde{\omega}=\lambda,
$$


where $\lambda=$ constant. This relation can be broken into two ordinary differential equations satisfied by the spatial and energy functions $Y$ and $H$. We obtain

$$
\begin{gathered}
\frac{1}{z^{2}} \frac{d}{d z}\left(z^{2} \frac{d Y}{d z}\right)+\lambda \eta^{2} Y=0 \\
\frac{1}{x^{2}} \frac{d}{d x}\left[x^{4}\left(H+\frac{d H}{d x}\right)\right]-\frac{s}{3 \Theta} H=0,
\end{gathered}
$$

where

$$
s \equiv \lambda-3 i \tilde{\omega}
$$

In the Fourier transform case under consideration here, the spatial function $Y$ must satisfy the mirror condition at the origin (cf. Equation (51)),

$$
\lim _{z \rightarrow 0} z^{2} \frac{d Y(\lambda, z)}{d z}=0
$$

Since Equation (69) is identical to Equation (46), which we previously encountered in Section 3.1 in our consideration of the time-averaged spectrum produced in a homogeneous spherical corona, we conclude that the fundamental solution for $Y$ is likewise given by (cf. Equation (52))

$$
Y(\lambda, z)=\frac{\sin (\eta z \sqrt{\lambda})}{\eta z}
$$

Furthermore, $Y$ must also satisfy the outer free-streaming boundary condition, and therefore the eigenvalues $\lambda_{n}$ are the roots of the equation (cf. Equation (153))

$$
\lim _{z \rightarrow 1}\left[\frac{1}{3 \eta} \frac{d Y(\lambda, z)}{d z}+Y(\lambda, z)\right]=0 .
$$

It follows that in a homogeneous corona, the Fourier eigenvalues $\lambda_{n}$ and spatial eigenfunctions $Y_{n}$ are exactly the same as those obtained in the treatment of the timeaveraged spectrum. Hence we can also conclude that the spatial eigenfunctions $Y_{n}$ form an orthogonal set, which motivates the development of a series expansion for the Fourier transformed radiation Green's function, $F_{\mathrm{G}}$. 
Comparison of Equations (70) and (47) allows us to immediately obtain the solution for the energy function $H$ as (cf. Equation (48))

$$
H(\lambda, x)=\left(x x_{0}\right)^{-2} e^{-\left(x+x_{0}\right) / 2} M_{2, \mu}\left(x_{\min }\right) W_{2, \mu}\left(x_{\max }\right)
$$

where $x_{\max }$ and $x_{\min }$ are defined in Equations (49), and

$$
\mu \equiv \sqrt{\frac{9}{4}+\frac{s}{3 \Theta}}=\sqrt{\frac{9}{4}+\frac{\lambda-3 i \tilde{\omega}}{3 \Theta}} .
$$

Following the same steps used in Section 3.1 for the development of the solution for the time-averaged radiation Green's function $f_{\mathrm{G}}^{\mathrm{S}}$, we can construct a series representation for the Fourier transform $F_{\mathrm{G}}$ by writing

$$
F_{\mathrm{G}}(x, z, \tilde{\omega})=\sum_{n=0}^{\infty} a_{n} H_{n}(x) Y_{n}(z),
$$

where the eigenfunctions $Y_{n}$ and $H_{n}$ are defined by

$$
Y_{n}(z) \equiv Y\left(\lambda_{n}, z\right), \quad H_{n}(x) \equiv H\left(\lambda_{n}, x\right)
$$

To solve for the expansion coefficients, $a_{n}$, we substitute Equation (177) into Equation (39) with $\alpha=0$ to obtain

$$
\lim _{\delta \rightarrow 0} \sum_{n=0}^{\infty} a_{n} Y_{n}(z)\left[H^{\prime}\left(x_{0}+\delta\right)-H^{\prime}\left(x_{0}-\delta\right)\right]=-\frac{N_{0} \delta\left(z-z_{0}\right) e^{i \tilde{\omega} p_{0}}}{4 \pi z_{0}^{2} x_{0}^{4} \Theta^{4}\left(m_{e} c^{2}\right)^{3} R^{3}},
$$

or, equivalently,

$$
\sum_{n=0}^{\infty} a_{n} Y_{n}(z) \mathscr{W}_{2, \mu}\left(x_{0}\right)=-\frac{N_{0} \delta\left(z-z_{0}\right) e^{i \tilde{\omega} p_{0}} e^{x_{0}}}{4 \pi z_{0}^{2} \Theta^{4}\left(m_{e} c^{2}\right)^{3} R^{3}}
$$

where the Wronskian is given by

$$
\mathscr{W}_{2, \mu}\left(x_{0}\right) \equiv M_{2, \mu}\left(x_{0}\right) W_{2, \mu}^{\prime}\left(x_{0}\right)-W_{2, \mu}\left(x_{0}\right) M_{2, \mu}^{\prime}\left(x_{0}\right)=-\frac{\Gamma(1+2 \mu)}{\Gamma(\mu-3 / 2)} .
$$

Substituting for the Wronskian in Equation (80) using Equation (81) and applying the operator $\int_{0}^{1} \eta^{3} z^{2} Y_{m}(z) d z$ to both sides of the equation, we can utilize the orthogonality of the spatial eigenfunctions $Y_{n}$ to obtain for the expansion coefficients $a_{n}$ the result

$$
a_{n}=\frac{N_{0} e^{i \tilde{\omega} p_{0}} e^{x_{0}} \eta^{3} \Gamma(\mu-3 / 2) Y_{n}\left(z_{0}\right)}{4 \pi \Theta^{4}\left(m_{e} c^{2}\right)^{3} R^{3} \Gamma(1+2 \mu) \mathscr{I}_{n}},
$$


where the quadratic normalization integrals $\mathscr{I}_{n}$ are defined in Equation (56).

By combining Equations (77) and (82), we find that the exact solution for the Fourier transformed radiation Green's function, $F_{\mathrm{G}}$, is given by the expansion

$$
F_{\mathrm{G}}(x, z, \tilde{\omega})=\frac{N_{0} e^{i \tilde{\omega} p_{0}} e^{x_{0}} \eta^{3}}{4 \pi R^{3} \Theta^{4}\left(m_{e} c^{2}\right)^{3}} \sum_{n=0}^{\infty} \frac{\Gamma(\mu-3 / 2)}{\Gamma(1+2 \mu) \mathscr{I}_{n}} Y_{n}\left(z_{0}\right) Y_{n}(z) H_{n}(x)
$$

with $\mu$ computed using Equation (76), and $Y_{n}$ and $H_{n}$ given by Equations (78). This result agrees with Equation (16) from KB once we note the change in the spatial variable from $z$ to $\tau=\eta z$, with $G_{n}(\tau)=Y_{n}(z), \tau_{*}=\eta$, and $\ell_{0}=R / \eta$. In the case of the exact solution for the time-averaged electron distribution derived in Section 3.1, we are able to derive an accurate approximation using asymptotic analysis (see Section 6.1.2). However, due to the complex nature of the series in Equation (83), it is not possible to extract useful asymptotic representations for the Fourier transform. Hence Equation (83) is the key result that will be utilized to compute the Fourier transform and the associated time lags for a spherical homogeneous cloud in Section 6.

\section{Inhomogeneous Model}

In the previous section, we have presented detailed solutions for the time-averaged spectrum and for the Fourier transform of the time-dependent photon Green's function describing the diffusion and Comptonization of photons in a spherical, homogeneous scattering cloud. Another interesting possibility is a coronal cloud with an electron number density distribution that varies as $n_{e}(r) \propto r^{-1}$, which was considered by HKC, and corresponds to $\alpha=1$ in Equations (16). In this case, the dimensionless radius $z$ is related to the scattering optical depth $\tau$ via (see Equations (27) and (28)

$$
\tau(z)=\eta \ln \left(z / z_{\text {in }}\right), \quad \tau_{*}=\eta \ln \left(1 / z_{\text {in }}\right)
$$


where $\tau_{*}$ is the optical thickness measured from the inner radius $r=r_{\text {in }}\left(z=z_{\text {in }}\right)$ to the outer radius $r=R(z=1)$. In this section, we obtain the analytical solutions for the time-averaged spectrum $f_{\mathrm{G}}^{\mathrm{S}}$ and for the Fourier transform $F_{\mathrm{G}}$ for the case with $n_{e}(r) \propto r^{-1}$.

\subsection{Quiescent Spectrum for $\alpha=1$}

The steady-state transport equation (32) describes the formation of the time-averaged X-ray spectrum via the thermal Comptonization of seed photons continually injected throughout a scattering corona with an electron number density profile given by $n_{e}(r) \propto r^{-\alpha}$. In the inhomogeneous case with $\alpha=1$, this equation can be solved using the separation form

$$
f_{\lambda}=K(\lambda, x) y(\lambda, z)
$$

to obtain, for $x \neq x_{0}$,

$$
\frac{-1}{y \eta^{2} z} \frac{d}{d z}\left(z^{3} \frac{d y}{d z}\right)=\frac{3 \Theta}{K x^{2}} \frac{d}{d x}\left[x^{4}\left(K+\frac{d K}{d x}\right)\right]=\lambda,
$$

where $\lambda=$ constant. The associated ordinary differential equations in the spatial and energy coordinates are, respectively,

$$
\begin{gathered}
\frac{1}{z} \frac{d}{d z}\left(z^{3} \frac{d y}{d z}\right)+\lambda \eta^{2} y=0 \\
\frac{1}{x^{2}} \frac{d}{d x}\left[x^{4}\left(K+\frac{d K}{d x}\right)\right]-\frac{\lambda}{3 \Theta} K=0 .
\end{gathered}
$$

Since Equation (88) is identical to Equation (47), it follows that the solution for the energy function $K$ is given by (cf. Equation (48))

$$
K(\lambda, x)=\left(x x_{0}\right)^{-2} e^{-\left(x+x_{0}\right) / 2} M_{2, \sigma}\left(x_{\min }\right) W_{2, \sigma}\left(x_{\max }\right)
$$

where

$$
\sigma \equiv \sqrt{\frac{9}{4}+\frac{\lambda}{3 \Theta}}
$$


The fundamental solutions for the spatial functions, $y$, are given by the power-law forms

$$
y(\lambda, z)=C_{1} z^{-1-\sqrt{1-\eta^{2} \lambda}}+z^{-1+\sqrt{1-\eta^{2} \lambda}},
$$

where $C_{1}$ is a superposition constant determined by applying the outer free-streaming boundary condition given by Equation (41). For the inhomogeneous case with $\alpha=1$, the outer boundary condition implies that $y$ must satisfy the equation

$$
\lim _{z \rightarrow 1}\left[\frac{z}{3 \eta} \frac{d y(\lambda, z)}{d z}+y(\lambda, z)\right]=0 .
$$

The corresponding result obtained for $C_{1}$ is

$$
C_{1}=\frac{3 \eta-1+\sqrt{1-\eta^{2} \lambda}}{1-3 \eta+\sqrt{1-\eta^{2} \lambda}} .
$$

The next step is to apply the inner free-streaming boundary condition, given by Equation (42). Stated in terms of $z$, we obtain for $\alpha=1$ the condition

$$
\lim _{z \rightarrow z_{\mathrm{in}}}\left[\frac{z}{3 \eta} \frac{d y(\lambda, z)}{d z}-y(\lambda, z)\right]=0,
$$

where $z_{\text {in }}=r_{\text {in }} / R$ is the dimensionless inner radius of the cloud. Equation (94) is satisfied only for certain discrete values of $\lambda$, which are the eigenvalues $\lambda_{n}$. The eigenvalues obtained are all positive real numbers. The resulting global functions $y$ therefore satisfy both the inner and outer free-streaming boundary conditions. Once the eigenvalues $\lambda_{n}$ are determined, the corresponding spatial and energy eigenfunctions are defined by

$$
y_{n}(z) \equiv y\left(\lambda_{n}, z\right), \quad K_{n}(x) \equiv K\left(\lambda_{n}, x\right)
$$

We show in Appendix A that the spatial eigenfunctions $y_{n}$ form an orthogonal set with respect to the weight function $z$, so that

$$
\int_{z_{\mathrm{in}}}^{1} z y_{n}(z) y_{m}(z) d z=0, \quad n \neq m
$$


We can therefore express the steady-state photon Green's function $f_{\mathrm{G}}^{\mathrm{S}}$ using the expansion

$$
f_{\mathrm{G}}^{\mathrm{S}}\left(x, x_{0}, z\right)=\sum_{n=0}^{\infty} c_{n} K_{n}(x) y_{n}(z)
$$

To solve for the expansion coefficients, $c_{n}$, we substitute Equation (97) into Equation (34), with $\alpha=1$, to obtain

$$
\lim _{\delta \rightarrow 0} \sum_{n=0}^{\infty} c_{n} y_{n}(z)\left[K_{n}^{\prime}\left(x_{0}+\delta\right)-K_{n}^{\prime}\left(x_{0}-\delta\right)\right]=-\frac{\dot{N}_{0}}{2 \pi R^{2} \eta c \Theta^{4}\left(m_{e} c\right)^{3} x_{0}^{4}\left(1-z_{\text {in }}^{2}\right)} .
$$

Eliminating $K$ using Equation (48) yields

$$
\sum_{n=0}^{\infty} c_{n} y_{n}(z) \mathscr{W}_{2, \sigma}\left(x_{0}\right)=-\frac{\dot{N}_{0} e^{x_{0}}}{2 \pi R^{2} \eta c \Theta^{4}\left(m_{e} c\right)^{3}\left(1-z_{\mathrm{in}}^{2}\right)},
$$

where the Wronskian $\mathscr{W}_{2, \sigma}\left(x_{0}\right)$ is defined in Equation (61). By combining Equations (99) and (62) we obtain

$$
\sum_{n=0}^{\infty} c_{n} y_{n}(z) \frac{\Gamma(1+2 \sigma)}{\Gamma(\sigma-3 / 2)}=\frac{\dot{N}_{0} e^{x_{0}}}{2 \pi R^{2} \eta c \Theta^{4}\left(m_{e} c\right)^{3}\left(1-z_{\text {in }}^{2}\right)}
$$

We can exploit the orthogonality of the spatial basis functions $y_{n}(z)$ with respect to the weight function $z$ by operating on Equation (100) with $\int_{z_{\text {in }}}^{1} z y_{m}(z) d z$ to obtain

$$
c_{n}=\frac{\dot{N}_{0} e^{x_{0}} \Gamma(\sigma-3 / 2) \mathscr{L}_{n}}{2 \pi R^{2} \eta c \Theta^{4}\left(m_{e} c^{2}\right)^{3} \mathscr{J}_{n} \Gamma(1+2 \sigma)\left(1-z_{\text {in }}^{2}\right)},
$$

where we have made the definitions

$$
\mathscr{J}_{n} \equiv \int_{z_{\mathrm{in}}}^{1} z y_{n}^{2}(z) d z, \quad \mathscr{L}_{n} \equiv \int_{z_{\mathrm{in}}}^{1} z y_{n}(z) d z
$$

The final result for the steady-state (quiescent) photon Green's function in the inhomogeneous case with $\alpha=1$ is obtained by combining Equations (97) and (101), which yields

$$
f_{\mathrm{G}}^{\mathrm{S}}\left(x, x_{0}, z\right)=\frac{\dot{N}_{0} e^{x_{0}}}{2 \pi R^{2} \eta c \Theta^{4}\left(m_{e} c^{2}\right)^{3}} \sum_{n=0}^{\infty} \frac{\Gamma(\sigma-3 / 2) \mathscr{L}_{n}}{\mathscr{J}_{n} \Gamma(1+2 \sigma)\left(1-z_{\mathrm{in}}^{2}\right)} K_{n}(x) y_{n}(z)
$$


with $\sigma$ computed using Equation (90), and $y_{n}$ and $K_{n}$ given by Equations (95). The timeaveraged X-ray spectrum computed using Equation (103) is compared with observational data for two specific sources in Section 6.1, and an accurate asymptotic approximation is derived in Section 6.1.2.

\subsection{Fourier Transform for $\alpha=1$}

In the inhomogeneous case with $\alpha=1$, we can substitute for the Fourier transform in Equation (38) using the separation functions

$$
F_{\lambda} \equiv K(\lambda, x) g(\lambda, z)
$$

to obtain, for $x \neq x_{0}$,

$$
-\frac{1}{g} \frac{1}{\eta^{2} z} \frac{d}{d z}\left(z^{3} \frac{d g}{d z}\right)-3 i \tilde{\omega} z=\frac{3 \Theta}{K x^{2}} \frac{d}{d x}\left[x^{4}\left(K+\frac{d K}{d x}\right)\right]=\lambda,
$$

where $\lambda$ is the separation constant. This relation yields two ordinary differential equations satisfied by the spatial and energy functions $g$ and $K$, given by

$$
\begin{aligned}
& \frac{1}{z} \frac{d}{d z}\left(z^{3} \frac{d g}{d z}\right)+\eta^{2}(\lambda+3 i \tilde{\omega} z) g=0 \\
& \frac{1}{x^{2}} \frac{d}{d x}\left[x^{4}\left(K+\frac{d K}{d x}\right)\right]-\frac{\lambda}{3 \Theta} K=0 .
\end{aligned}
$$

Equation (107) is identical to Equation (47), and therefore we can immediately conclude that the solution for the energy function $K$ is given by

$$
K(\lambda, x)=\left(x x_{0}\right)^{-2} e^{-\left(x+x_{0}\right) / 2} M_{2, \sigma}\left(x_{\min }\right) W_{2, \sigma}\left(x_{\max }\right)
$$

where

$$
\sigma \equiv \sqrt{\frac{9}{4}+\frac{\lambda}{3 \Theta}}
$$


One significant new feature in the inhomogeneous case with $\alpha=1$ under consideration here is that the eigenvalues $\lambda_{n}$ are now functions of the Fourier frequency $\tilde{\omega}$, which stems from the appearance of $\tilde{\omega}$ in Equation (106). It follows that $\sigma$ is also a function of $\tilde{\omega}$ through its dependence on $\lambda$ (see Equation (50) ). This inconvenient mixing of variables forces us to generate a separate list of eigenvalues for each sampled frequency. The fundamental solution for the spatial function $g$ is given by the superposition

$$
g(\lambda, z)=\frac{1}{z}\left[C_{2} J_{-\nu}(2 \eta \sqrt{3 i \tilde{\omega} z})+J_{\nu}(2 \eta \sqrt{3 i \tilde{\omega} z})\right]
$$

where $J_{\nu}(z)$ denotes the Bessel function of the first kind, and we have made the definition

$$
\nu \equiv 2 \sqrt{1-\eta^{2} \lambda}
$$

The superposition constant $C_{2}$ is computed by applying the outer free-streaming boundary condition, which can be written as (cf. Equation (92))

$$
\lim _{z \rightarrow 1}\left[\frac{z}{3 \eta} \frac{d g(\lambda, z)}{d z}+g(\lambda, z)\right]=0
$$

The result obtained for $C_{2}$ is

$$
C_{2}=\frac{(2-6 \eta+\nu) J_{\nu}(2 \eta \sqrt{3 i \tilde{\omega}})-2 \eta \sqrt{3 i \tilde{\omega}} J_{\nu-1}(2 \eta \sqrt{3 i \tilde{\omega}})}{(6 \eta-2+\nu) J_{-\nu}(2 \eta \sqrt{3 i \tilde{\omega}})+2 \eta \sqrt{3 i \tilde{\omega}} J_{-\nu-1}(2 \eta \sqrt{3 i \tilde{\omega}})}
$$

Next we must apply the inner free-streaming boundary condition given by (cf. Equation (94))

$$
\lim _{z \rightarrow z_{\mathrm{in}}}\left[\frac{z}{3 \eta} \frac{d g(\lambda, z)}{d z}-g(\lambda, z)\right]=0
$$

where $z_{\text {in }}=r_{\text {in }} / R$. The roots of Equation (114) are the eigenvalues $\lambda_{n}$, and the associated global functions $g$ satisfy both the inner and outer free-streaming boundary conditions. The corresponding spatial and energy eigenfunctions are given by

$$
g_{n}(z) \equiv g\left(\lambda_{n}, z\right), \quad K_{n}(x) \equiv K\left(\lambda_{n}, x\right)
$$


As demonstrated in Appendix A, the spatial eigenfunctions $g_{n}$ are orthogonal with respect to the weight function $z$, and therefore

$$
\int_{z_{\mathrm{in}}}^{1} z g_{n}(z) g_{m}(z) d z=0, \quad n \neq m
$$

It follows that we can express the Fourier transformed radiation Green's function, $F_{\mathrm{G}}$, using the expansion (cf. Equation (77))

$$
F_{\mathrm{G}}(x, z, \omega)=\sum_{n=0}^{\infty} d_{n} K_{n}(x) g_{n}(z)
$$

The expansion coefficients $d_{n}$ can be computed by applying the derivative jump condition given by Equation (39), which yields for $\alpha=1$

$$
\left.\lim _{\delta \rightarrow 0}\left[\frac{d F_{\mathrm{G}}}{d x}\right]\right|_{x_{0}-\delta} ^{x_{0}+\delta}=-\frac{N_{0} \delta\left(z-z_{0}\right) e^{i \tilde{\omega} p_{0}}}{4 \pi x_{0}^{4} z_{0}^{2} z^{-1} \Theta^{4}\left(m_{e} c^{2}\right)^{3} R^{3}} .
$$

Combining Equations (117) and (118) gives the result

$$
\lim _{\delta \rightarrow 0} \sum_{n=0}^{\infty} d_{n} g_{n}(z)\left[K^{\prime}\left(x_{0}+\delta\right)-K^{\prime}\left(x_{0}-\delta\right)\right]=-\frac{N_{0} \delta\left(z-z_{0}\right) e^{i \tilde{\omega} p_{0}}}{4 \pi x_{0}^{4} z_{0}^{2} z^{-1} \Theta^{4}\left(m_{e} c^{2}\right)^{3} R^{3}},
$$

or, equivalently,

$$
\sum_{n=0}^{\infty} d_{n} g_{n}(z) \mathscr{W}_{2, \sigma}\left(x_{0}\right)=\sum_{n=0}^{\infty}-d_{n} g_{n}(z) \frac{\Gamma(1+2 \sigma)}{\Gamma(\sigma-3 / 2)}=-\frac{N_{0} \delta\left(z-z_{0}\right) e^{i \tilde{\omega} p_{0}} e^{x_{0}}}{4 \pi z_{0}^{2} z^{-1} \Theta^{4}\left(m_{e} c^{2}\right)^{3} R^{3}}
$$

where we have utilized Equations (61) and (62) for the Wronskian $\mathscr{W}_{2, \sigma}\left(x_{0}\right)$.

We can solve for the expansion coefficients $d_{n}$ by utilizing the orthogonality of the spatial eigenfunctions $g_{n}$ with respect to the weight function $z$. Applying $\int_{z_{\mathrm{in}}}^{1} z g_{m}(z) d z$ to both sides of Equation (120), we obtain, after some algebra,

$$
d_{n}=\frac{N_{0} e^{i \tilde{\omega} p_{0}} e^{x_{0}} \Gamma(\sigma-3 / 2) g_{n}\left(z_{0}\right)}{4 \pi \Theta^{4}\left(m_{e} c^{2}\right)^{3} R^{3} \Gamma(1+2 \sigma) \mathscr{K}_{n}},
$$

where the quadratic normalization integrals, $\mathscr{K}_{n}$, are defined by

$$
\mathscr{K}_{n} \equiv \int_{z_{\text {in }}}^{1} z g_{n}^{2}(z) d z
$$


The final result for the Fourier transform $F_{\mathrm{G}}$ of the photon Green's function $f_{\mathrm{G}}$ obtained by combining Equations (117) and (121) is

$$
F_{\mathrm{G}}(x, z, \tilde{\omega})=\frac{N_{0} e^{i \tilde{\omega} p_{0}} e^{x_{0}}}{4 \pi R^{3} \Theta^{4}\left(m_{e} c^{2}\right)^{3}} \sum_{n=0}^{\infty} \frac{\Gamma(\sigma-3 / 2)}{\Gamma(1+2 \sigma) \mathscr{K}_{n}} g_{n}\left(z_{0}\right) g_{n}(z) K_{n}(x),
$$

with $\sigma$ evaluated using Equation (109), and $g_{n}$ and $K_{n}$ given by Equations (115). This exact analytical solution can be used to generate theoretical predictions of the Fourier transformed data streams in two different energy channels in order to simulate the time lags created in a spherical scattering corona with an electron number density profile that varies as $n_{e}(r) \propto r^{-1}$. As in the case of the homogeneous Fourier transform discussed in Section 3.2, it is not possible to extract useful asymptotic representations for the inhomogeneous Fourier transform due to the complex nature of the sum appearing in Equation (123).

\section{Bremsstrahlung Injection}

The investigations carried out by Miyamoto (1988), HKC, and KB show that the impulsive injection of monochromatic seed photons into a homogeneous Comptonizing corona cannot produce the observed dependence of the X-ray time lags on the Fourier frequency. A major advantage of the analytical method we employ here is that the radiation Green's function we obtain can be convolved with any desired seed photon distribution as a function of radius $r$, energy $\epsilon$, and time $t$. This flexibility stems from the fact that the transport equation is a linear partial differential equation. A source spectrum of particular interest is a flash of bremsstrahlung seed photons injected on a spherical shell at radius $r=r_{0}$. We may expect the observed variability in this case to be qualitatively different from the behavior associated with a monochromatic flash of seed photons, because the bremsstrahlung flash represents broadband radiation. We anticipate that the prompt escape of high-energy photons from the bremsstrahlung seed distribution may cause a profound shift in the dependence of the observed X-ray time lags on the Fourier period. 
Since the fundamental transport equation governing the radiation field is linear, it follows that we can compute the time-dependent spectrum $f$ resulting from any seed photon distribution $Q$ that is an arbitrary function of time, energy, and radius using the integral convolution

$$
f(\epsilon, r, t)=\int_{0}^{\infty} \int_{r_{\mathrm{in}}}^{R} \int_{0}^{\infty} 4 \pi r_{0}^{2} \epsilon_{0}^{2} f_{\mathrm{G}}\left(\epsilon, \epsilon_{0}, r, r_{0}, t, t_{0}\right) Q\left(\epsilon_{0}, r_{0}, t_{0}\right) N_{0}^{-1} d \epsilon_{0} d r_{0} d t_{0}
$$

where $4 \pi r_{0}^{2} \epsilon_{0}^{2} Q\left(\epsilon_{0}, r_{0}, t_{0}\right) d r_{0} d t_{0} d \epsilon_{0}$ gives the number of photons injected in the energy range $d \epsilon_{0}$, radius range $d r_{0}$, and time range $d t_{0}$ around the coordinates $\left(\epsilon_{0}, r_{0}, t_{0}\right)$. In the case of optically thin bremsstrahlung injection, the seed photons are created as a result of a local instability in the coronal plasma, due to, for example, a magnetic reconnection event, or the passage of a shock. It follows that the photon distribution resulting from localized, impulsive injection of bremsstrahlung radiation at radius $r=r_{0}$ can be written as

$$
f_{\mathrm{brem}}(\epsilon, r, t)=\int_{\epsilon_{\mathrm{abs}}}^{\infty} f_{\mathrm{G}}\left(\epsilon, \epsilon_{0}, r, r_{0}, t, t_{0}\right) Q_{\mathrm{brem}}\left(\epsilon_{0}\right) N_{0}^{-1} d \epsilon_{0}
$$

where $\epsilon_{\mathrm{abs}}$ denotes the low-energy cutoff due to free-free self-absorption in the source plasma, and the bremsstrahlung source function, $Q_{\mathrm{brem}}$, for fully-ionized hydrogen is given by (Rybicki \& Lightman 1979)

$$
Q_{\text {brem }}\left(\epsilon_{0}\right)=\frac{A_{0}}{\epsilon_{0}} e^{-\epsilon_{0} / k T_{e}}
$$

where

$$
A_{0}=\frac{2^{5} \pi q^{6}}{3 h m_{e} c^{3}}\left(\frac{2 \pi}{3 k m_{e}}\right)^{1 / 2} V_{0} t_{\mathrm{rad}} T_{e}^{-1 / 2} n_{e}^{2}\left(r_{0}\right)
$$

Here, $V_{0}$ denotes the radiating volume, $t_{\text {rad }}$ is the radiating time interval, and $q$ is the electron charge. The bremsstrahlung source function is normalized so that $Q_{\text {brem }}\left(\epsilon_{0}\right) d \epsilon_{0}$ gives the number of photons injected in the energy range between $\epsilon_{0}$ and $\epsilon_{0}+d \epsilon_{0}$.

The low-energy self-absorption cutoff, $\epsilon_{\mathrm{abs}}$, appearing in Equation (125), depends on the temperature and density of the plasma experiencing the transient that produces the 
flash of bremsstrahlung seed photons. The density of the unstable plasma is expected to be higher than that in the surrounding corona, due to either shock compression or a thermal instability. We do not analyze this physical process in detail here, and instead we treat $\epsilon_{\text {abs }}$ as a free parameter in our model, although a more detailed physical picture could be developed in future work.

Changing variables from $(\epsilon, r, t)$ to $(x, z, p)$ and applying Fourier transformation to both sides of Equation (125), we obtain

$$
F_{\text {brem }}\left(x, z, z_{0}, \tilde{\omega}\right)=A_{0} N_{0}^{-1} \int_{x_{\mathrm{abs}}}^{\infty} F_{\mathrm{G}}\left(x, x_{0}, z, z_{0}, \tilde{\omega}\right) x_{0}^{-1} e^{-x_{0}} d x_{0}
$$

where $x_{\mathrm{abs}}=\epsilon_{\mathrm{abs}} /\left(k T_{e}\right)$ is the dimensionless self-absorption energy. The function $F_{\mathrm{G}}$ in Equation (128) represents the Fourier transformation of the time-dependent photon Green's function for either the homogeneous or inhomogeneous cases, given by either Equation (83) or Equation (123), respectively. The integral with respect to $x_{0}$ can be carried out analytically, and the exact solutions are given by

$F_{\text {brem }}\left(x, z, z_{0}, \tilde{\omega}\right)=\frac{e^{i \tilde{\omega} p_{0}} \eta^{3} A_{0} e^{-x / 2}}{4 \pi R^{3} \Theta^{4}\left(m_{e} c^{2}\right)^{3} x^{2}} \sum_{n=0}^{\infty} \begin{cases}\frac{\Gamma(\mu-3 / 2) Y_{n}\left(z_{0}\right) Y_{n}(z)}{\Gamma(1+2 \mu) \mathscr{I}_{n}} B(\mu, x), & \text { homogeneous } \\ \frac{\Gamma(\sigma-3 / 2) g_{n}\left(z_{0}\right) g_{n}(z)}{\Gamma(1+2 \sigma) \eta^{3} \mathscr{K}_{n}} B(\sigma, x), & \text { inhomogeneous }\end{cases}$

where $\sigma$ and $\mu$ are given by Equations (150) and (76), respectively, and the integral function $B(\lambda, x)$ is defined by

$$
B(\lambda, x) \equiv \int_{x_{\mathrm{abs}}}^{\infty} e^{-x_{0} / 2} x_{0}^{-3} M_{2, \lambda}\left(x_{\min }\right) W_{2, \lambda}\left(x_{\max }\right) d x_{0} .
$$

We show in Appendix B that $B(\lambda, x)$ can be evaluated analytically to obtain the closed-form result

$$
B(\lambda, x)= \begin{cases}W_{2, \lambda}(x)\left[I_{M}(\lambda, x)-I_{M}\left(\lambda, x_{\mathrm{abs}}\right)\right]-M_{2, \lambda}(x) I_{W}(\lambda, x), & x \geq x_{\mathrm{abs}} \\ -M_{2, \lambda}(x) I_{W}\left(\lambda, x_{\mathrm{abs}}\right), & x \leq x_{\mathrm{abs}}\end{cases}
$$


where the functions $I_{M}$ and $I_{W}$ are defined by

$$
I_{M}(\lambda, x) \equiv \frac{x^{-2} e^{-x / 2}}{\lambda+\frac{3}{2}}\left(M_{1, \lambda}(x)+\frac{3}{\lambda+\frac{1}{2}}\left\{M_{0, \lambda}(x)+\frac{2}{\lambda-\frac{1}{2}}\left[M_{-1, \lambda}(x)+\frac{1}{\lambda-\frac{3}{2}} M_{-2, \lambda}(x)\right]\right\}\right)
$$

and

$$
I_{W}(\lambda, x) \equiv x^{-2} e^{-x / 2}\left[-W_{1, \lambda}(x)+3 W_{0, \lambda}(x)-6 W_{-1, \lambda}(x)+6 W_{-2, \lambda}(x)\right] .
$$

Section 6, we will use this result to study the implications of broadband (bremsstrahlung) seed photon injection as an alternative to monochromatic injection for the production of the observed X-ray time lags in homogeneous and inhomogeneous scattering coronae.

\section{Astrophysical Applications}

In the previous sections, we have obtained the exact mathematical solution for the steady-state photon Green's function, $f_{\mathrm{G}}^{\mathrm{S}}$, describing the X-ray emission emerging from a scattering corona as a result of the continual distributed injection of monochromatic seed photons. We have also obtained the exact solution for the Green's function, $F_{\mathrm{G}}$, describing the Fourier transform of the X-ray spectrum resulting from the impulsive localized injection of monochromatic seed photons into the corona. By convolving the solution for $F_{\mathrm{G}}$ with the bremsstrahlung source term, we were also able to derive the exact solution for the bremsstrahlung Fourier transform, $F_{\text {brem }}$.

The availability of these various solutions for the steady-state X-ray spectrum and for the Fourier transform resulting from impulsive injection allows us to explore a wide variety of injection scenarios, while maintaining explicit control over the physical parameters describing the astrophysical objects of interest, such as the temperature, the electron number density, and the cloud radius. Our goal here is to develop "integrated models," in which the coupled calculations of the time-averaged X-ray spectrum and the transient 
Fourier X-ray time lags are based on the same set of physical parameters (temperature, density, radius) for the scattering corona. We believe that this integrated approach represents a significant step forward by facilitating the study of a broad range of parameter space using an analytical model.

\subsection{Comparison with Observed Time-Averaged Spectra}

The time-averaged X-ray spectrum emanating from the outer surface of the cloud results from the continual distributed injection of soft photons from a source with a rate that is proportional to the local electron number density. Thus, there is no specific injection radius for the time-averaged model. The detailed solutions we have obtained describe the radiative transfer occurring in either a homogeneous cloud, or in an inhomogeneous cloud in which the electron number density varies with radius as $n_{e}(r) \propto r^{-1}$.

Application of the integrated model begins with a comparison of the observed time-averaged X-ray spectrum with the theoretical steady-state photon flux measured at the detector, $\mathcal{F}_{\epsilon}(\epsilon)$, computed using the relation

$$
\mathcal{F}_{\epsilon}(\epsilon)=\left.\left(\frac{R}{D}\right)^{2} c \epsilon^{2} f_{\mathrm{G}}^{\mathrm{S}}\left(\frac{\epsilon}{k T_{e}}, x_{0}, z\right)\right|_{z=1},
$$

where $D$ is the distance to the source, $R$ is the radius of the corona, $c$ is the speed of light, and the solution for the steady-state spectrum, $f_{\mathrm{G}}^{\mathrm{S}}\left(x, x_{0}, 1\right)$, at the surface of the cloud is evaluated using either Equation (66) for the homogeneous case or Equation (103) for the inhomogeneous case. In our computations of the time-averaged X-ray spectra, the seed photon energy is frozen at $\epsilon_{0}=0.1 \mathrm{keV}$ in order to approximate the effect of the continual injection of blackbody photons from a "cool" accretion disk with temperature $T \sim 10^{6} \mathrm{~K}$.

The temperature parameter $\Theta=k T_{e} / m_{e} c^{2}$ (Equation (201)) and the scattering parameter $\eta=R / \ell_{*}$ (Equation (22) ) determine the slope of the power-law component 
of the time-averaged spectrum, and also the frequency of the high-energy exponential cutoff created by recoil losses. In the inhomogeneous case, the shape of the time-averaged spectrum also depends on the dimensionless inner radius, $z_{\text {in }}=r_{\text {in }} / R$, at which the inner free-streaming boundary condition is imposed. We vary the values of $\Theta, \eta$, and $z_{\text {in }}$ until good qualitative agreement with the shape of the observed steady-state X-ray spectrum is achieved. Once the values of $\Theta, \eta$, and $z_{\text {in }}$ are determined, the photon injection rate, $\dot{N}_{0}$, is then computed by matching the theoretical flux level with the observed time-averaged spectrum.

\subsubsection{Exact Time-Averaged X-ray Spectra}

In Figure 1, we plot the theoretical time-averaged (quiescent) X-ray spectra measured at the detector, $\mathcal{F}_{\epsilon}(\epsilon)$, computed using the homogenous corona model, with distributed seed photon injection, evaluated by combining Equations (66) and (134). The plots also include a comparison with the observed X-ray spectra for Cyg X-1 and GX 339-04. The data for Cyg X-1 were reported by Cadolle Bel et al. (2006) and cover the observation period MJD 52617-52620, and the data for GX 339-04 were reported by Cadolle Bel et al. (2011) and cover the observation period MJD 55259.9-55261.1. Both sources were observed by INTEGRAL in the low/hard state. The model parameters are summarized in Table 1, and the corresponding homogeneous eigenvalues are plotted in Figure 3. The time-averaged X-ray spectra obtained for the inhomogeneous corona model, computed by combining Equations (103) and (134), are plotted and compared with the observational data in Figure 2, and the corresponding inhomogeneous eigenvalues are depicted in Figure 3.

We find that the observed time-averaged spectra can be fit equally well using either the homogeneous or inhomogeneous cloud models. Furthermore, the homogeneous and 
inhomogeneous models have similar temperatures and cloud radii. This behavior illustrates the fact that the time-averaged spectrum mainly depends on the cloud temperature and the Compton $y$-parameter, and is not directly dependent on the accretion geometry, as discussed in detail by Sunyaev \& Titarchuk (1980).
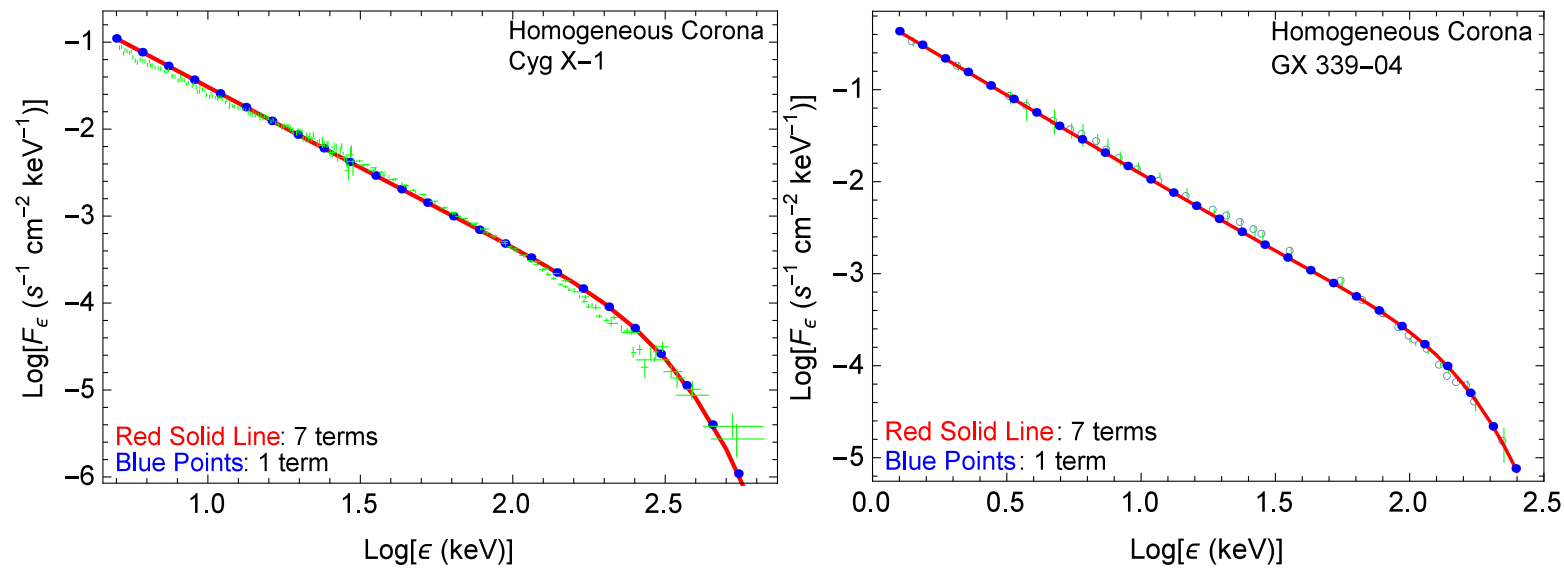

Fig. 1.- : Theoretical time-averaged (quiescent) X-ray spectra, $\mathcal{F}_{\epsilon}(\epsilon)$, observed at the detector, for a homogeneous corona, with constant electron number density, $n_{e}$, computed by combining Equations (66) and (134). Results are presented for Cyg X-1 (left panel) and GX 339-04 (right panel), along with observational data taken from Cadolle Bel et al. (2006) and Cadolle Bel et al. (2011), respectively. Both sources were observed in the low/hard state using INTEGRAL. To analyze the convergence of the series, we plot the results obtained using only the first term in the series, or using the first 7 terms. The convergence is extremely rapid for both sources.

It is interesting to compare our model parameters with those used by HKC, who computed the time-averaged spectra of Cyg X-1 for a variety of electron density profiles, similar to the homogeneous and inhomogeneous cloud configurations studied here. They employed a scattering cloud with a homogeneous central region, coupled with either a 

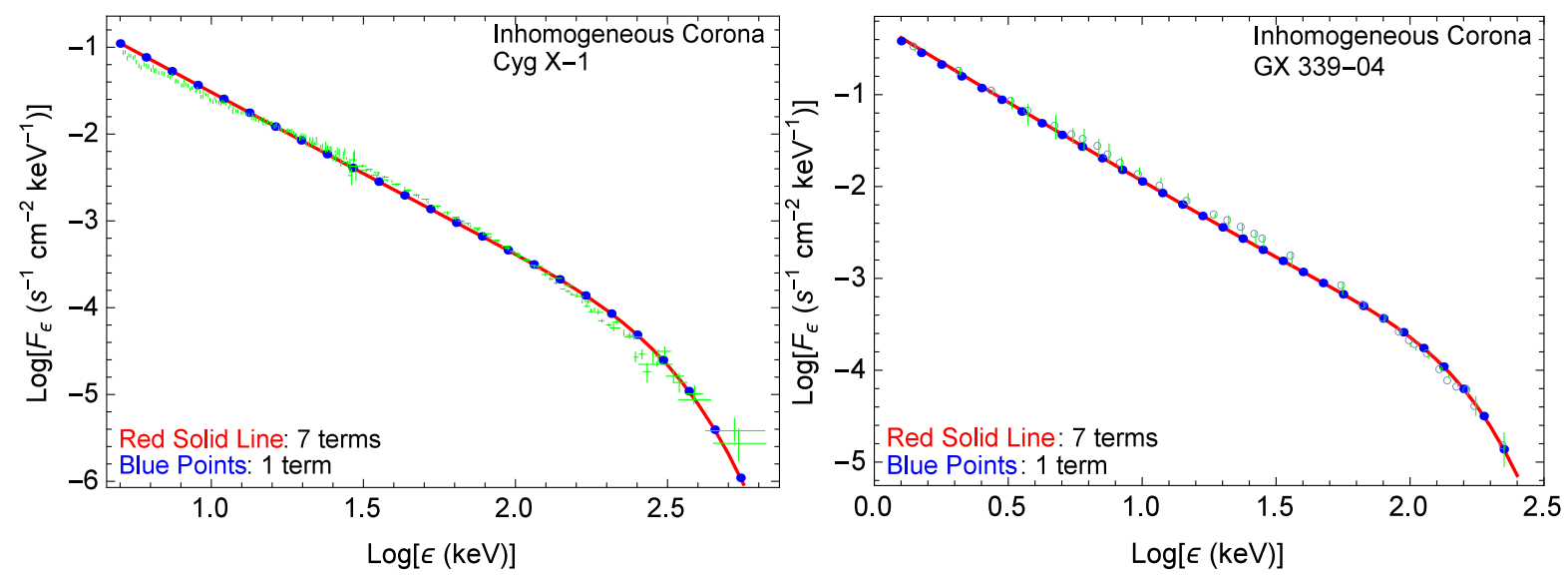

Fig. 2.- : Same as Figure 1, except we plot the time-averaged X-ray spectra emanating from an inhomogeneous corona, with electron density profile $n_{e}(r) \propto r^{-1}$. The results were obtained by combining Equations (103) and (134). The convergence is very rapid.
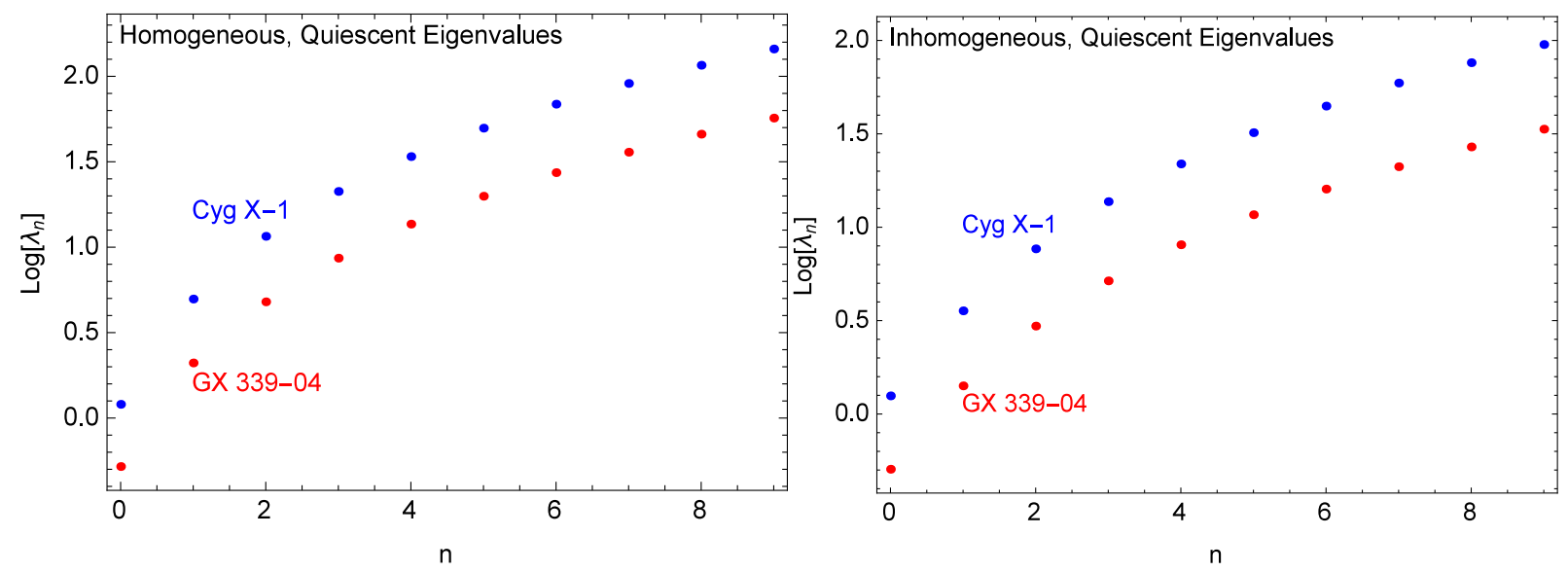

Fig. 3.- : Real eigenvalues, $\lambda_{n}$, for the time-averaged (quiescent) spectrum radiated by a homogeneous corona (left panel), and an inhomogeneous corona (right panel). All of the eigenvalues are positive. 
homogeneous or inhomogeneous outer region. The HKC cloud has a scattering optical thickness $\tau_{*}=1$ and an electron temperature of $k T_{e}=100 \mathrm{keV}$, whereas we obtain $\tau_{*} \sim 2-3$ and $k T_{e} \sim 60 \mathrm{keV}$ (see Table 1). The differences between our model parameters and theirs could be due to the fact that the observational data analyzed here corresponds to the low/hard state of Cyg X-1, whereas HKC compared their model with spectral data from Ling et al. (1997), acquired while Cyg X-1 was in its high/soft state, when the source is known to have a lower optical depth (e.g., Frontera et al. 2001; Malzac 2012; Del Santo et al. 2013). Furthermore, the values of $\tau_{*}$ and $T_{e}$ that we obtain are very close to those found by Malzac et al. (2008), who also considered the low/hard state of Cyg X-1.

In Table 2 we compare the energy injection rate for the seed photons in our model, $L_{\text {inj }}$, with the time-averaged X-ray luminosity, $L_{\mathrm{X}}$, observed in the low/hard state for the two sources studied here, Cyg X-1 and GX 339-04. The injection luminosity is computed using $L_{\text {inj }}=\epsilon_{0} \dot{N}_{0}$, where $\dot{N}_{0}$ is the photon injection rate and the seed photon energy is $\epsilon_{0}=0.1 \mathrm{keV}$. The values for $L_{\mathrm{X}}$ were taken from Cadolle Bel et al. (2006) for Cyg X-1, and from Cadolle Bel et al. (2011) for GX 339-04. We see that the injection luminosity is $\sim 10 \%$ of the observed X-ray luminosity, which is consistent with the values we have obtained for the effective Compton $y$-parameter.

Table 1. Input Model Parameters

\begin{tabular}{clcccccccc}
\hline \hline Source & \multicolumn{1}{c}{ Model } & $\eta$ & $\Theta$ & $k T_{e}(\mathrm{keV})$ & $\epsilon_{\mathrm{abs}}(\mathrm{keV})$ & $z_{\text {in }}$ & $z_{0}$ & $t_{*}(\mathrm{~s})$ & $\tau_{*}$ \\
\hline \multirow{2}{*}{ Cyg X-1 } & Homogeneous & 2.50 & 0.120 & 61.3 & 1.60 & 0.00 & 1.00 & 0.040 & 2.50 \\
Cyg X-1 & Inhomogeneous & 1.40 & 0.122 & 62.4 & 1.60 & 0.12 & 0.91 & 0.065 & 2.97 \\
GX 339-04 & Homogeneous & 4.00 & 0.064 & 32.7 & 0.01 & 0.00 & 0.78 & 0.038 & 4.00 \\
GX 339-04 & Inhomogeneous & 2.20 & 0.064 & 32.7 & 0.01 & 0.10 & 0.60 & 0.090 & 5.07 \\
\hline
\end{tabular}




\subsubsection{Approximate Power-Law X-ray Spectra}

The X-ray spectra plotted in Figures 1 and 2 have a power-law form that extends up to the exponential cutoff, where electron recoil losses become significant. This suggests the existence of an approximate, asymptotic power-law solution, valid in the domain $x \lesssim 1$ (Rybicki \& Lightman 1979). Figures 1 and 2 also include a convergence study, where we compare the results obtained for the steady-state spectra, $f_{\mathrm{G}}^{\mathrm{S}}$, using only the first $(n=0)$ term in the series with the fully-converged result obtained using the first 7 terms in the series. The results are essentially indistinguishable, which establishes that the convergence of the series is extremely rapid. The power-law shape observed for $x \lesssim 1$, combined with the rapid convergence, suggest that we can derive an asymptotic power-law solution by analyzing the first term in the expansion for the observed flux. By analogy with previous work on thermal Comptonization, we expect that the properties of the approximate analytical solution will shed light on the relationship between the first eigenvalue, $\lambda_{0}$, which determines the spectral slope, and the effective Compton $y$-parameter for the model. We derive the approximate asymptotic power-law solution below, for both the homogeneous and inhomogeneous cloud configurations.

We are interested in photon energies well above the injection energy, $\epsilon_{0}=0.1 \mathrm{keV}$, and therefore it follows that $x>x_{0}$. In this case, we can combine Equations (48) and (66) to

Table 2. Auxiliary Model Parameters

\begin{tabular}{|c|c|c|c|c|c|c|c|c|c|}
\hline Source & Model & $\dot{N}_{0}\left(\mathrm{~s}^{-1}\right)$ & $L_{\mathrm{inj}}\left(\operatorname{ergs~s}^{-1}\right)$ & $L_{\mathrm{X}}\left(\operatorname{ergs~s}^{-1}\right)$ & $y_{\text {eff }}$ & $\tau_{\text {eff }}$ & $\lambda_{0}$ & $R(\mathrm{~cm})$ & $D(\mathrm{kpc})$ \\
\hline Cyg X-1 & Homogeneous & $2.00 \times 10^{46}$ & $3.20 \times 10^{36}$ & $2.20 \times 10^{37}$ & 1.20 & 1.58 & 1.20 & $3.00 \times 10^{9}$ & 2.4 \\
\hline Cyg X-1 & Inhomogeneous & $2.70 \times 10^{46}$ & $4.33 \times 10^{36}$ & $2.20 \times 10^{37}$ & 1.17 & 1.55 & 1.25 & $2.73 \times 10^{9}$ & 2.4 \\
\hline GX 339-04 & Homogeneous & $5.75 \times 10^{46}$ & $9.21 \times 10^{36}$ & $6.28 \times 10^{37}$ & 1.48 & 2.40 & 0.52 & $4.56 \times 10^{9}$ & 8.0 \\
\hline GX 339-04 & Inhomogeneous & $7.00 \times 10^{46}$ & $1.12 \times 10^{37}$ & $6.28 \times 10^{37}$ & 1.51 & 2.43 & 0.51 & $5.94 \times 10^{9}$ & 8.0 \\
\hline
\end{tabular}


express the time-averaged X-ray spectrum in the homogeneous corona as

$$
f_{\mathrm{G}}^{\mathrm{S}}\left(x, x_{0}, z\right)=\frac{9 \dot{N}_{0} e^{\left(x_{0}-x\right) / 2}\left(x x_{0}\right)^{-2}}{4 \pi R^{2} c \Theta^{4}\left(m_{e} c^{2}\right)^{3}} \sum_{n=0}^{\infty} \frac{\Gamma(\sigma-3 / 2) \sin \left(\eta \sqrt{\lambda_{n}}\right)}{\lambda_{n} \Gamma(1+2 \sigma) \mathscr{I}_{n}} Y_{n}(z) M_{2, \sigma}\left(x_{0}\right) W_{2, \sigma}(x) .
$$

The corresponding result obtained by combining Equations (89) and (103) in the inhomogenous case is

$$
f_{\mathrm{G}}^{\mathrm{S}}\left(x, x_{0}, z\right)=\frac{\dot{N}_{0} e^{\left(x_{0}-x\right) / 2}\left(x x_{0}\right)^{-2}}{2 \pi R^{2} \eta c \Theta^{4}\left(m_{e} c^{2}\right)^{3}} \sum_{n=0}^{\infty} \frac{\Gamma(\sigma-3 / 2) \mathscr{L}_{n}}{\mathscr{J}_{n} \Gamma(1+2 \sigma)\left(1-z_{\text {in }}^{2}\right)} y_{n}(z) M_{2, \sigma}\left(x_{0}\right) W_{2, \sigma}(x) .
$$

Based on Figure 1, we observe that the domain of the power-law shape is $x_{0}<x \lesssim 1$. This suggests that we can employ Equations (13.1.32), (13.1.33), (13.5.5), and (13.5.6) from Abramowitz \& Stegun (1970) to implement the small-argument asymptotic form for the Whittaker functions $M$ and $W$.

We will only evaluate the $n=0$ term in the sum, since it represents a converged result, according to the results plotted in Figure 1. After some algebra, the approximate solution obtained in the homogeneous case is

$$
f_{\mathrm{G}}^{\mathrm{S}}\left(x, x_{0}, z\right) \approx \frac{9 \dot{N}_{0} x_{0}^{\sigma_{0}-3 / 2}}{8 \pi R^{2} c \Theta^{4}\left(m_{e} c^{2}\right)^{3}} \frac{\sin \left(\eta \sqrt{\lambda_{0}}\right)}{\lambda_{0} \sigma_{0} \mathscr{I}_{0}} \frac{\sin \left(\eta z \sqrt{\lambda_{0}}\right)}{\eta z} x^{-\sigma_{0}-3 / 2},
$$

where (see Equation (50))

$$
\sigma_{0} \equiv \sqrt{\frac{9}{4}+\frac{\lambda_{0}}{3 \Theta}}
$$

Likewise, in the inhomogeneous case, we obtain

$$
f_{\mathrm{G}}^{\mathrm{S}}\left(x, x_{0}, z\right) \approx \frac{\dot{N}_{0} x_{0}^{\sigma_{0}-3 / 2}}{4 \pi R^{2} \eta c \Theta^{4}\left(m_{e} c^{2}\right)^{3}} \frac{\mathscr{L}_{0} y_{0}(z)}{\mathscr{J}_{0} \sigma_{0}\left(1-z_{\text {in }}^{2}\right)} x^{-\sigma_{0}-3 / 2}
$$

By substituting either Equation (137) or (139) into Equation (134), and setting $z=1$, we can compute the corresponding approximate X-ray spectrum, $\mathcal{F}_{\epsilon}(\epsilon)$, observed at the detector. These results are plotted and compared with the exact solutions in Figure 4, and it is clear that the power-law approximation is extremely accurate below the exponential cutoff energy, as expected. 
We can obtain further insight into the physical significance of our approximate power-law solutions by comparing our work with previous results. First, we note that within the regime of interest here, $x \lesssim 1$, and therefore electron recoil losses are negligible. This suggests that we can define an effective $y$-parameter by comparing our work with the corresponding analytical solutions that neglect recoil losses. This situation was treated by Rybicki \& Lightman (1979), who obtained power-law solutions to the Kompaneets equation by utilizing an escape-probability formalism for the spatial photon transport, as an alternative to the spatial diffusion operator employed here. In our solutions, given by Equations (137) and (139), the power-law index is equal to $-\sigma_{0}-3 / 2$. Setting our result equal to the index $m$ given by Equation (7.76) from Rybicki \& Lightman (1979) yields

$$
-\sigma_{0}-\frac{3}{2}=-\frac{3}{2}-\sqrt{\frac{9}{4}+\frac{4}{y_{\mathrm{eff}}}},
$$

where $y_{\text {eff }}$ is the effective Compton $y$-parameter and $\Theta$ is the dimensionless temperature ratio. Using Equation (138) to substitute for $\sigma_{0}$ and solving for $y_{\text {eff }}$, we find that

$$
y_{\text {eff }}=\frac{12 \Theta}{\lambda_{0}}
$$

The values obtained for $y_{\text {eff }}$ and $\lambda_{0}$ in our calculations of the time-averaged X-ray spectra resulting from distributed (density-weighted) seed photon injection are reported in Table 2 . We generally find that $y_{\text {eff }} \sim 1$, corresponding to unsaturated Comptonization, which is consistent with the power-law spectra plotted in Figures 1 and 2 (e.g., Sunyaev \& Titarchuk $1980)$.

It is also interesting to relate the first eigenvalue, $\lambda_{0}$, to the effective optical depth, $\tau_{\text {eff }}$, traversed by the photons as they propagate through the scattering corona, and ultimately escape. Referring to the simplified escape-probability model analyzed by Rybicki \& Lightman (1979), we can apply their Equation (7.41) to write, in the optically thick case,

$$
y=4 \Theta \tau_{\text {eff }}^{2}
$$


Setting $y=y_{\text {eff }}$ and combining Equations (141) and (142), we find that $\tau_{\text {eff }}$ and $\lambda_{0}$ are related via

$$
\tau_{\text {eff }}=\sqrt{\frac{3}{\lambda_{0}}} .
$$

The results obtained for $\tau_{\text {eff }}$ are listed in Table 2. Comparing the values of $\tau_{\text {eff }}$ with the values for $\tau_{*}$ in Table 1 , we conclude that $\tau_{\text {eff }} \sim 0.5 \tau_{*}$, which reflects the fact that the seed photon injection is density weighted, rather than being localized at the center of the cloud. Hence, on average, photons traverse less optical depth than is given by $\tau_{*}$, which is measured from the cloud center.
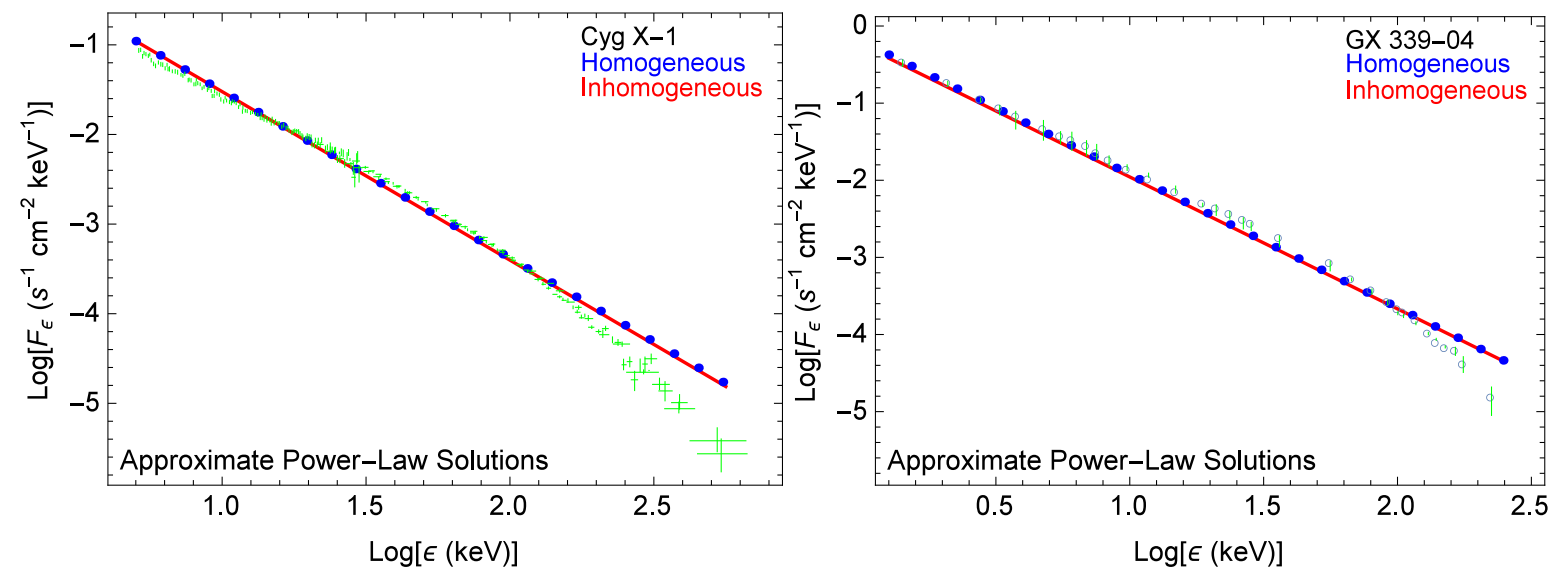

Fig. 4.-: Approximate power-law X-ray spectra, $\mathcal{F}_{\epsilon}(\epsilon)$, computed using Equation (134) combined with Equation (137) for the homogeneous corona (blue filled circles) or Equation (139) for the inhomogeneous corona (red solid lines). The results are compared with the observational data for Cyg X-1 (left panel) and GX 339-04 (right panel). See the discussion in the text. 


\subsection{Comparison with Time Lag Data}

In the time-dependent case, the time lags are computed using the Fourier transforms evaluated at the surface of the cloud, after an impulsive localized transient injects seed photons with a specified spectrum at a specific radius. This represents a sudden, low-luminosity flash of radiation that subsequently scatters and Comptonizes throughout the cloud before the final signal escapes to the observer.

The theoretical prediction for the time lag observed between hard channel energy $\epsilon_{\text {hard }}$ and soft channel energy $\epsilon_{\text {soft }}$ at Fourier frequency $\nu_{f}$ is computed using the van der Klis et al. (1987) formula (cf. Equation (4)),

$$
\delta t=\frac{\arg \left[S^{*}\left(x_{\mathrm{soft}}, \tilde{\omega}\right) H\left(x_{\mathrm{hard}}, \tilde{\omega}\right)\right]}{2 \pi \nu_{f}},
$$

where the dimensionless energies $x_{\text {soft }}$ and $x_{\text {hard }}$ are defined by

$$
x_{\mathrm{soft}} \equiv \frac{\epsilon_{\mathrm{soft}}}{k T_{e}}, \quad x_{\mathrm{hard}} \equiv \frac{\epsilon_{\mathrm{hard}}}{k T_{e}} .
$$

The Fourier transforms of the soft and hard channel time series are computed using

$$
S\left(x_{\mathrm{soft}}, \tilde{\omega}\right)=F\left(x_{\mathrm{soft}}, \tilde{\omega}\right), \quad H\left(x_{\mathrm{hard}}, \tilde{\omega}\right)=F\left(x_{\mathrm{hard}}, \tilde{\omega}\right)
$$

where $F$ represents the Fourier transform radiated at the surface of the coronal cloud, at radius $r=R(z=1)$. We assume that the observed time lags are the result of the time-dependent Comptonization of seed photons injected with either a monochromatic or bremsstrahlung initial energy distribution. Our results for the homogeneous and inhomogeneous Fourier transforms in the case of monochromatic photon injection are given by Equations (83) and (123), respectively, and our results for the homogeneous and inhomogeneous Fourier transforms in the case of bremsstrahlung injection are both

covered by Equation (129). In the case of bremsstrahlung injection, we must also impose a low-energy self-absorption cutoff at energy $\epsilon=\epsilon_{\text {abs }}$ in order to avoid producing an infinite number of seed photons. 
All of our analytical formulas for the Fourier transform are expressed in terms of the dimensionless Fourier frequency, $\tilde{\omega}$, which is related to the dimensional Fourier frequency, $\nu_{f}$, measured in $\mathrm{Hz}$, via (see Equation (37))

$$
\tilde{\omega}=2 \pi \nu_{f} t_{*}
$$

where the scattering time, $t_{*}=\ell_{*} / c$, is equal to the mean-free time at the outer edge of the cloud. The value of $t_{*}$ is related to the cloud radius $R$ and the value of $\eta$ via (see Equation (22))

$$
t_{*}=\frac{\ell_{*}}{c}=\frac{R}{\eta c} .
$$

Once the values for the temperature parameter $\Theta$, the scattering parameter $\eta$, and the inner radius $z_{\text {in }}$ have been tied down via comparison of the observed time-averaged spectrum with the theoretical steady-state spectrum for a given source, the next step is to vary the values of the cloud radius, $R$, and the bremsstrahlung self-absorption energy, $\epsilon_{\text {abs }}$, until we achieve reasonable qualitative agreement between the theoretical time lags and the observed time lags. This allows us to translate between the dimensionless Fourier frequency $\tilde{\omega}$ and the dimensional frequency $\nu_{f}$ using Equation (147), with the scattering time $t_{*}$ computed using Equation (148). We consider several different scenarios for the calculation of the X-ray time lags below and compare the results with the observational data for Cyg X-1 and GX 339-04.

\subsubsection{Monochromatic Injection in Inhomogeneous Corona}

When the injected spectrum is monochromatic, or nearly so, and the injection takes place in a homogeneous cloud, all of the authors who have examined the problem agree that the resulting time lags are independent of Fourier frequency, in contradiction to the observations (e.g. Miyamoto 1988, HKC, KB). Hence it is interesting to explore the 
consequences of altering the cloud configuration in our model to treat monochromatic seed photon injection in an inhomogeneous corona, with electron number density distribution $n_{e}(r) \propto r^{-1}$, which was also considered by HKC. Since the injected seed photons are monochromatic, with energy $\epsilon_{0}=0.1 \mathrm{keV}$, we must use the Fourier transform Green's function, $F_{\mathrm{G}}$, to compute the time lags by combining Equations (123), (144), and (146). The time lags resulting from monochromatic injection in an inhomogeneous cloud are plotted as a function of the Fourier frequency $\nu_{f}$ and compared with the Cyg X-1 data from Nowak et al. (1999) in Figure 5 for both large and small cloud radii. The channel energy values used are $\epsilon_{\text {soft }}=2 \mathrm{keV}$ and $\epsilon_{\text {hard }}=11 \mathrm{keV}$, which correspond to the channel-center energies used in the analysis of the observational data. It is clear that the model results do not fit the data very well for either value of the cloud radius. Note that the shape of the time lag curves exhibits the same trend as the data, but the magnitude is too large. This is a result of the long upscattering time required for the soft disk seed photons to reach the soft and hard channel energies.

HKC also computed time lags for monochromatic injection in an inhomogeneous cloud, but they were able to fit the observational data, in contrast to our results. However, in order to qualitatively match the observed time lags, HKC had to adopt an outer cloud radius of $\sim 1$ light-second $\left(3 \times 10^{10} \mathrm{~cm}\right)$, which is an order of magnitude larger than the cloud radii implied by our model. The discrepancy between the model results may be due to the fact that their cloud is optically thin, whereas our cloud is optically thick. The values for the optical depth derived here are consistent with those obtained during the low/hard state of Cyg X-1 by Malzac et al. (2008), Malzac (2012), Del Santo et al. (2013), and Frontera et al. (2001). Unfortunately, we can't use our model to explore the region of parameter space studied by HKC because the corona must be optically thick in order to justify the diffusion approximation employed in our approach. 


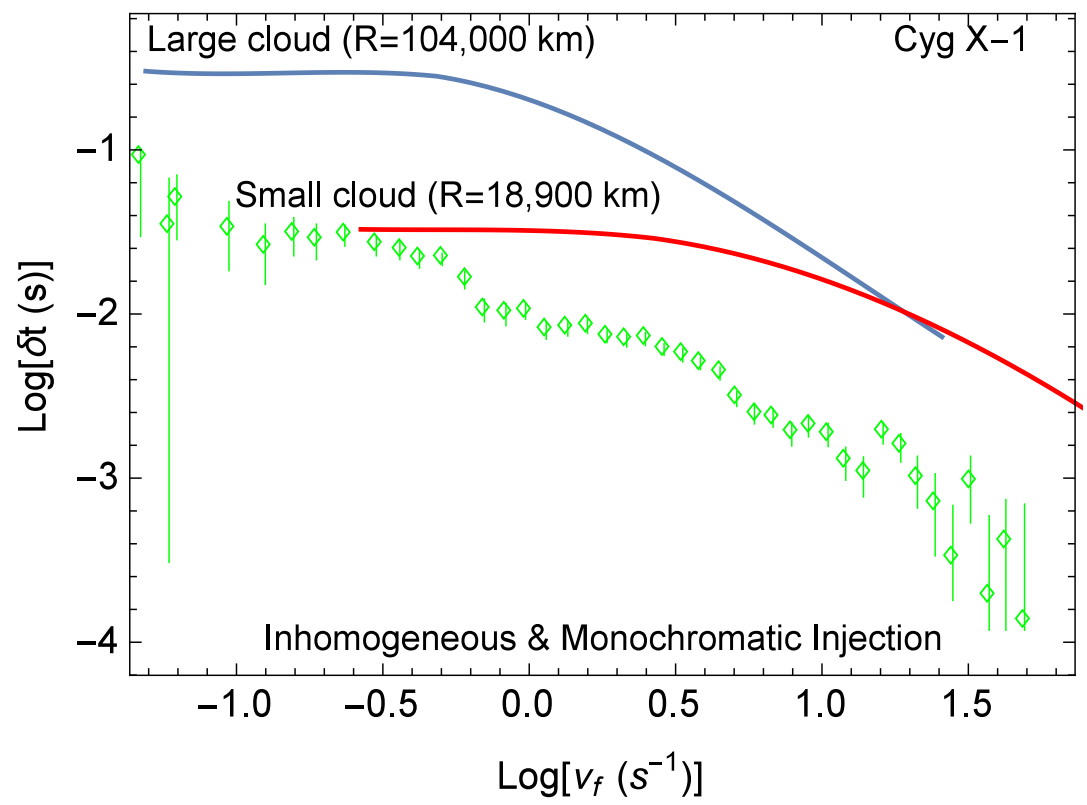

Fig. 5.-: Theoretical time lag profiles resulting from monochromatic injection in an inhomogeneous cloud, with electron number density profile $n_{e}(r) \propto r^{-1}$, compared with the Cyg X-1 time lag data from Nowak et al. (1999). The source was in the low/hard state during the observation. The time lags are computed by combining Equations (123), (144), and (146), and the channel energies used in the theoretical calculations are $\epsilon_{\text {soft }}=2 \mathrm{keV}$ and $\epsilon_{\text {hard }}=11 \mathrm{keV}$. The photon injection energy is $\epsilon_{0}=0.1 \mathrm{keV}$.

\subsubsection{Variation of Seed Photon Distribution}

It is apparent from Figure 5 that monochromatic injection into an inhomogeneous corona is unable to generate good agreement with the time lag data. Furthermore, it has been previously established by Miyamoto (1988), HKC, and KB that monochromatic injection into a homogeneous cloud also fails to agree with the data. Hence, it is interesting to use our new formalism to explore the alternative hypothesis of broadband (bremsstrahlung) seed photon injection, rather than monochromatic injection.

The bremsstrahlung-injection time lags are computed by combining Equations (129), 
(144), and (146), and the model parameters are varied until reasonable qualitative agreement with the observational data is achieved. We plot the theoretical bremsstrahlung-injection time lags as a function of the Fourier frequency $\nu_{f}$ in Figure 6, using both the homogeneous and inhomogeneous coronal cloud models. The results are compared with the observational data for Cyg X-1 and GX 339-04 taken from Nowak et al. (1999) and Cassatella et al. (2012), respectively. The corresponding physical parameters are listed in Table 1, and the channel energies used in the theoretical calculations are $\epsilon_{\text {soft }}=2 \mathrm{keV}$ and $\epsilon_{\text {hard }}=11 \mathrm{keV}$ for Cyg X-1, and $\epsilon_{\text {soft }}=2 \mathrm{keV}$ and $\epsilon_{\text {hard }}=10 \mathrm{keV}$ for GX 339-04, which correspond to the channel-center energies used in the observational calculations of the time lags. The low-energy self-absorption cutoff is set at $\epsilon_{\mathrm{abs}}=1.6 \mathrm{keV}$ for Cyg X-1 and at $\epsilon_{\mathrm{abs}}=0.01 \mathrm{keV}$ for GX 339-04. In the case of the homogeneous corona, the eigenvalues $\lambda_{n}$ for the Fourier transform solution are the same real values obtained in the analysis of the time-averaged (quiescent) spectrum, which are plotted in the left-hand panel in Figure 3. In the case of the inhomogeneous corona, the eigenvalues $\lambda_{n}$ are complex, and are plotted in Figure 7 .

We find that in order to match the observational time lag data, the impulsive injection of the bremsstrahlung photons must occur near the outer edge of the cloud, with $z_{0} \lesssim 1$. The transient that produces the soft seed photons is not treated in detail here, but we note that the outer edge of the corona is a region which the disk suddenly expands in the vertical direction, possibly leading to various types of plasma instabilities. In particular, the abrupt change in magnetic topology may generate rapid reconnection events that can result in the injection of a significant population of soft seed photons via bremsstrahlung emission (e.g., Poutanen \& Fabian 1999).

In contrast with the behavior of the monochromatic injection scenario studied by Miyamoto (1988), the results depicted in Figure 6 show that in the case of broadband (bremsstrahlung) seed photon injection into either a homogeneous or inhomogeneous cloud, 
Comptonization can produce Fourier frequency-dependent time lags that agree with the observational data for both Cyg X-1 and GX 339-04. The diminishing time lags at high Fourier frequency are explained as a natural results of the prompt escape of broadband seed photons, combined with the delayed escape of upscattered Comptonized photons over longer timescales.
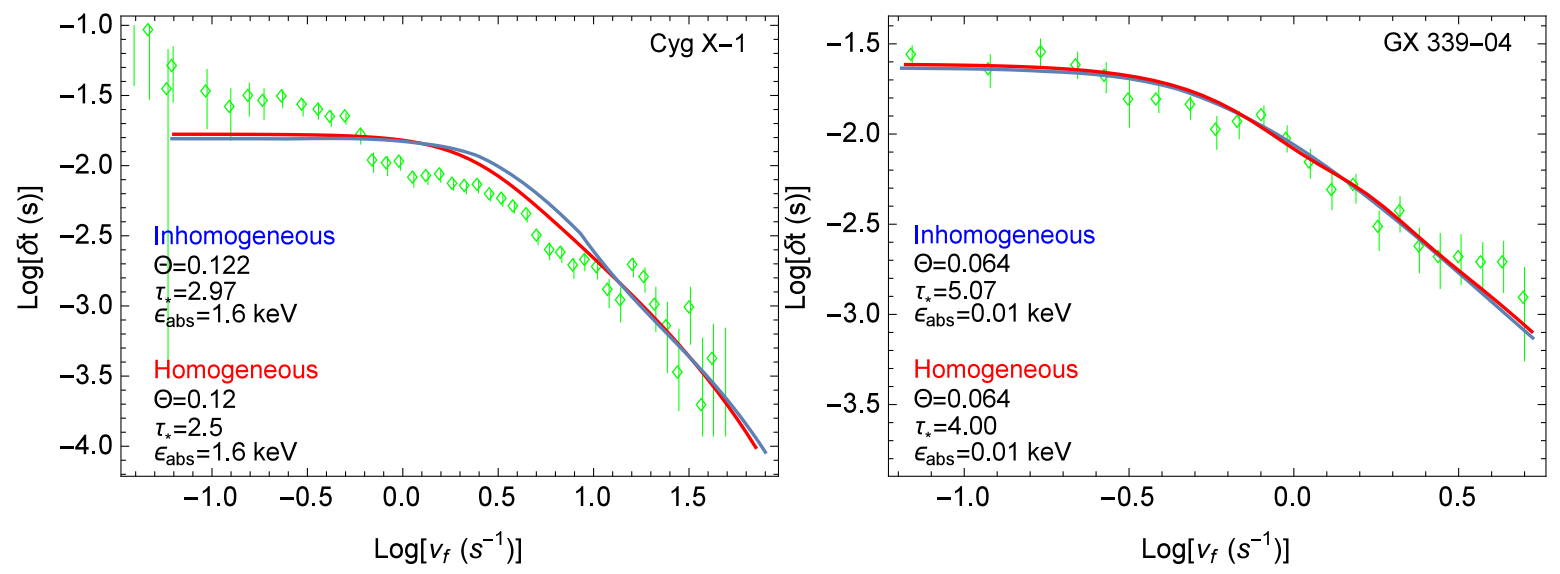

Fig. 6.- : Theoretical time lag profiles for bremsstrahlung seed photon injection in a homogeneous corona (red) and an inhomogeneous corona (blue), compared with the data for Cyg X-1 (left panel) from Nowak et al. (1999), and the data for GX 339-04 (right panel) from Cassatella et al. (2012). Each source was observed in the low/hard state. See Section 6.2.3 and Figure 8 for a discussion of the convergence properties.

This indicates that the critical quantities for determining the shape of the time-lag profile are the overall optical thickness of the cloud and its temperature, which have nearly the same values in the homogeneous and inhomogeneous corona models. We therefore conclude that the actual configuration of the cloud (i.e. the detailed radial variation of the electron number density) is not well constrained by either the observations of the time lags or the observations of the time-averaged X-ray spectrum, and indeed, either cloud configuration works equally well, although there is a slight difference in the resulting cloud 

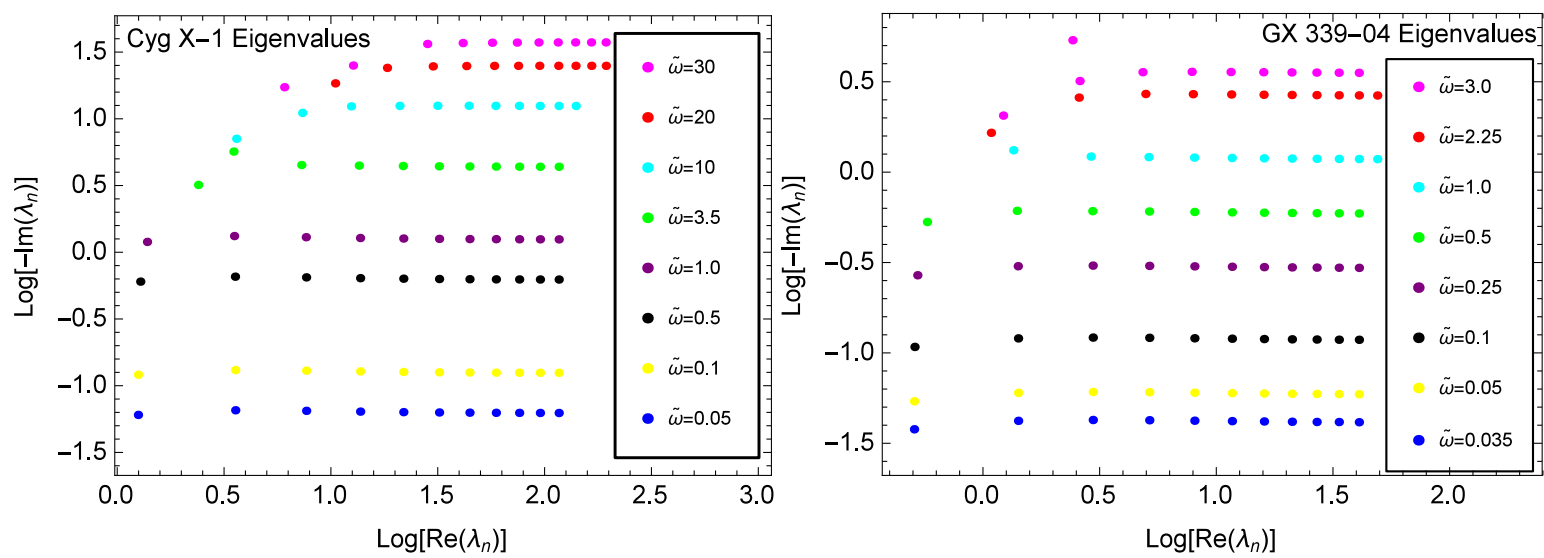

Fig. 7.- : Complex eigenvalues, $\lambda_{n}$, for the Fourier transform in the inhomogeneous case. Left panel is for Cyg X-1 and right panel is for GX 339-04. Note that the imaginary part of $\lambda_{n}$ is always negative, and therefore we change the sign before taking the log. The colors refer to the indicated values of the dimensionless Fourier frequency $\tilde{\omega}$, and the sequences running from left to right represent the values of $\lambda_{0}$ through $\lambda_{10}$.

radius $R$, as indicated in Table 2 .

\subsubsection{Convergence of Time Lags}

In our model, the time lags are computed based on analytical expressions for the Fourier transform of the emitted radiation spectrum. Since these expressions are stated in terms of series expansions, it is important to examine the convergence of the results obtained for the time lags as one increases the truncation level of the series. Obviously, rapid smooth convergence is desirable.

In Figure 8, we present a convergence study of the theoretical time lags computed using the models for Cyg X-1 and GX 339-04, based on both the homogeneous and 
inhomogeneous cloud configurations. In each panel, the black curves represent the time lags evaluated using only the first term in the expansions, and the red and blue curves represent fully converged results, where no significant change will occur upon the addition of another term. The red and blue curves are the same as the final results for the time lags plotted in Figure 6. The time lags generally require about 20 terms to fully converge, whereas the expansions for the time-averaged spectra converge immediately (see Figures 1 and 2).
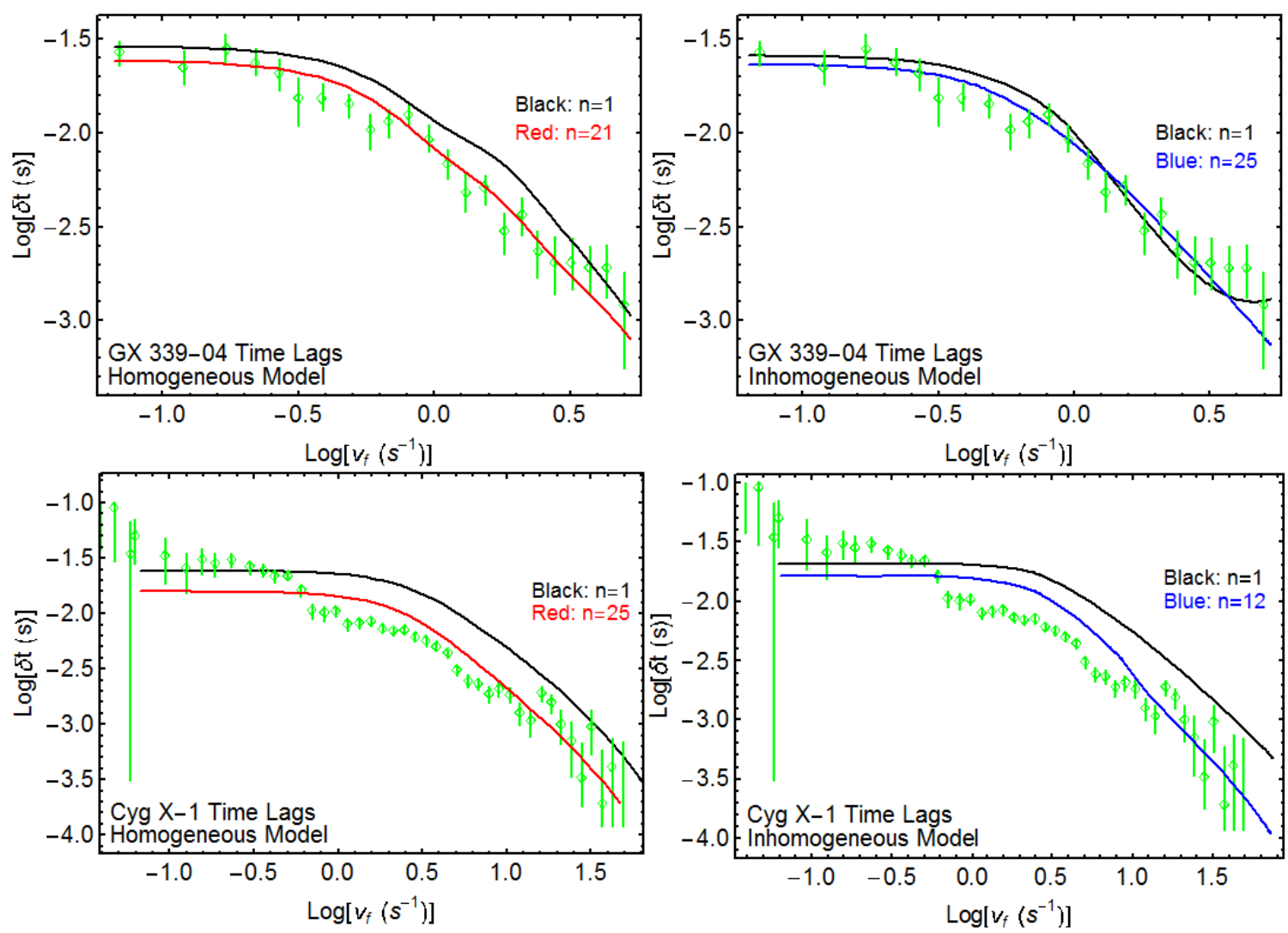

Fig. 8.-: Convergence study of the theoretical time lags for Cyg X-1 and GX 339-04 computed using either the homogeneous or the inhomogeneous cloud model. The number of terms used in the series expansions for the Fourier transforms is indicated for each curve. The red and blue curves correspond to the final results plotted in Figure 6. 


\section{Discussion and Conclusion}

We have obtained the exact analytical solution for the problem of time-dependent thermal Comptonization in a spherical scattering corona, based on two different electron density profiles. By working in the Fourier domain, we have obtained a closed-form expression for the Green's function corresponding to the injection of monochromatic seed photons into a cloud at a single radius and time. The radiated Fourier transform, evaluated at the surface of the cloud, can be directly substituted into the time lag formula introduced by van der Klis et al. (1987) in order to compute the predicted dependence of the lags on the Fourier frequency for any selected X-ray channel energies. In our approach, the time-averaged X-ray spectrum and the time lags are both computed using the same set of physical parameters to describe the properties of the scattering cloud, and therefore our formalism represents an integrated model that fully describes the high-energy spectral and timing properties of the source.

\subsection{Relation to Previous Work}

The study presented by HKC is similar to ours, although their methodology and input assumptions are somewhat different. HKC focused exclusively on a single injection scenario, namely the injection of essentially monoenergetic, low-temperature blackbody seed photons at the center of the scattering cloud. Based on this injection spectrum, they concluded that the observed time lag behavior in Cyg X-1 could not be reproduced unless the electron number density profile was inhomogeneous, with $n_{e}(r) \propto r^{-1}$ for example. In this case, although the predicted time lags fit the observed dependence on the Fourier period, the resulting dimensions of the cloud are so large that the requisite heating is difficult to accomplish based on any of the standard dissipation models. 
Another notable difference between the work of HKC and the results developed here is that we have obtained a set of exact mathematical solutions, whereas HKC utilized a numerical Monte Carlo simulation method. This distinction is important, because by exploiting the exact solution for the Fourier transform of the Green's function, we are able to explore a much wider range of injection scenarios, in which we can vary both the location of the initial flash of seed photons, and its spectral distribution. Based on our analytical formalism, we are able to confirm the results of HKC regarding monochromatic injection, but we have also generalized those results by exploring the implications of varying the seed photon injection radius and spectrum. We find that the injection of broadband (bremsstrahlung) seed photons relatively close to the surface of a homogeneous or inhomogeneous cloud can fit the observed time lag profiles at least as well as the HKC model does, but with a cloud size an order of magnitude smaller. In Section 7.2 we discuss the physical reasons underlying the success of the bremsstrahlung injection scenario.

The treatment of electron scattering in our work differs from that utilized by HKC, since we have adopted the Thomson cross section, whereas HKC implemented the full expression for the Klein-Nishina cross section. In principle, utilization of the Klein-Nishina cross section would be expected to affect the hard time lags, due to the quantum reduction in the scattering probability at high energies. However, for the photon energy range of interest here, $\sim 0.1-10 \mathrm{keV}$, combined with our maximum electron temperature, $k T_{e}=62.4 \mathrm{keV}$, not many photons are likely to sample the reduced cross section, which requires an incident photon energy exceeding $500 \mathrm{keV}$ as seen in the rest frame of the electron. Hence it seems surprising that HKC observed a significant change in the normalization of their computed time lags when they adopted the Klein-Nishina cross section instead of the Thomson value. We suspect that this may be due to the somewhat higher electron temperature they used, $k T_{e}=100 \mathrm{keV}$. 
To explore this question quantitatively, we can compute the fraction of electrons such that an incident photon of a given energy in the lab frame exceeds $500 \mathrm{keV}$ in the electron's rest frame. The relevant thermal distribution function for the calculation is the relativistic Maxwell-Jüttner distribution, given by (e.g., Ter Haar \& Wergeland 1971; Hua 1997)

$$
f_{\mathrm{MJ}}(\gamma) \equiv \frac{\gamma \sqrt{\gamma^{2}-1}}{\Theta K_{2}(1 / \Theta)} \exp \left(-\frac{\gamma}{\Theta}\right)
$$

where $\Theta \equiv k T_{e} /\left(m_{e} c^{2}\right)$ and $K_{2}$ denotes the modified Bessel function of the second kind. The probability that a randomly-selected electron has a Lorentz factor in the range between $\gamma$ and $\gamma+d \gamma$ is equal to $f_{\mathrm{MJ}}(\gamma) d \gamma$.

In order to compute an upper bound on the probability of generating a scattering in the Klein-Nishina regime, we shall focus on the most energetic possible collision scenario, which is a head-on collision between the electron and the photon. In this case, the incident photon energy in the electron's rest frame, $E_{0}^{\prime}$, is given by

$$
E_{0}^{\prime}=E_{0}\left(\frac{1+\beta}{1-\beta}\right)^{1 / 2}, \quad \beta^{2}=1-\frac{1}{\gamma^{2}}
$$

where $E_{0}$ is the incident photon energy in the lab frame. By integrating the Maxwell-Jüttner distribution, we can compute the probability, $P$, that a randomly-selected electron has sufficient energy to create the required incident photon energy of at least $500 \mathrm{keV}$ in the rest frame. The probability is given by

$$
P=\int_{\gamma_{0}}^{\infty} f_{\mathrm{MJ}}(\gamma) d \gamma
$$

where the lower bound $\gamma_{0}$ is the root of the equation

$$
500 \mathrm{keV}=E_{0}\left(2 \gamma_{0}^{2}-1+2 \gamma_{0} \sqrt{\gamma_{0}^{2}-1}\right)^{1 / 2}
$$

Setting the incident photon energy $E_{0}=100 \mathrm{keV}$ as an extreme example, we find that the lower bound is $\gamma_{0}=2.6$. Adopting the $\mathrm{HKC}$ temperature value, $k T_{e}=100 \mathrm{keV}$, we 
obtain $\Theta=0.2$, in which case the probability given by Equation (151) is $P=3.1 \times 10^{-3}$. This probability may be large enough to explain the variation of the HKC time lag results observed when they switched between the Thomson cross section and the Klein-Nishina cross section, if some of the photons inverse-Compton scatter up to high enough energies to sample the Klein-Nishina regime, before returning to lower energies via Compton scattering. We can also compute the scattering probability $P$ based on the maximum electron temperature that we have adopted in our applications, $k T_{e}=62.4 \mathrm{keV}$, which yields $\Theta=0.122$. In this case, one finds that the Maxwell-Jüttner integration gives $P=2.6 \times 10^{-5}$, which is much smaller than the HKC result. Hence we conclude that utilization of the Klein-Nishina cross section would probably not make a significant difference in our applications. However, we can't reach any definitive conclusions about this question using the model developed here since it is based on the assumption of Thomson scattering in the electron's rest frame.

\subsection{Formation of the Light Curves}

The somewhat surprising difference between the time lag profiles produced when the injection spectrum has a monoenergetic shape versus a broadband shape can be explored by using the inverse Fourier transform to compute the time-dependent light curves for the hard and soft energy channels in the two cases. To accomplish this, we must make use of the inversion integral (cf. Equation (36) $)$

$$
f(x, z, p)=\frac{1}{2 \pi} \int_{-\infty}^{\infty} e^{-i \tilde{\omega} p} F(x, z, \tilde{\omega}) d \tilde{\omega},
$$

where $F$ is the Fourier transform computed using either the monochromatic injection Green's function solution (Equation (83) for the homogeneous cloud, or Equation (123)

for the inhomogeneous cloud), or the bremsstrahlung injection solution (the homogeneous and inhomogeneous cases are both computed using Equation (129)). Evaluation of 
Equation (153) requires numerical integration since the inversion integral cannot be performed analytically. We therefore focus on a few simple examples in order to illustrate the dependence of the light curves on the injection model.

In Figure 9, we plot the hard and soft channel light curves computed using Equation (153) for the case of a homogeneous cloud experiencing impulsive injection of either low-energy monochromatic seed photons or broadband (bremsstrahlung) seed photons. The parameters describing the monochromatic injection scenario are temperature $\Theta=0.12$, injection location $z_{0}=1$, injection energy $\epsilon_{0}=0.1 \mathrm{keV}$, soft channel energy $\epsilon_{\text {soft }}=2 \mathrm{keV}$, and hard channel energy $\epsilon_{\text {hard }}=10 \mathrm{keV}$. In the case of bremsstrahlung injection, we set $\Theta=0.12, z_{0}=1, \epsilon_{\mathrm{abs}}=0.1 \mathrm{keV}, \epsilon_{\mathrm{soft}}=2 \mathrm{keV}$, and $\epsilon_{\mathrm{soft}}=10 \mathrm{keV}$. One can immediately identify the characteristic Fast Rise Exponential Decay (FRED) shape (e.g., Sunyaev \& Titarchuk 1980) for each channel signal. As expected, the hard channel curve is delayed in time relative to the soft channel curve due to upscattering, but the detailed relationship between the two light curves depends qualitatively on whether the injection spectrum is monochromatic or broadband.

One clearly observes that the two FRED curves resulting from monochromatic injection in a homogeneous cloud are of the same shape, and are simply shifted by a perfect delay with respect to one another on all timescales (see Figure 9). This yields a constant time lag across all Fourier frequencies (or periods), in agreement with the Miyamoto result that $\mathrm{HKC}$ and $\mathrm{KB}$ have confirmed. Our physical understanding of this behavior is as follows. Since all of the initial photons start with the same energy in the monochromatic case, the time lag is purely a result of Compton reverberation, where the upscattering timescale is proportional to the logarithm of the ratio of the hard to soft energies (Payne 1980). Based on this simple example, we conclude that monochromatic injection anywhere in a homogeneous cloud cannot produce Fourier frequency-dependent time lags, in contradiction 
with the observational data.
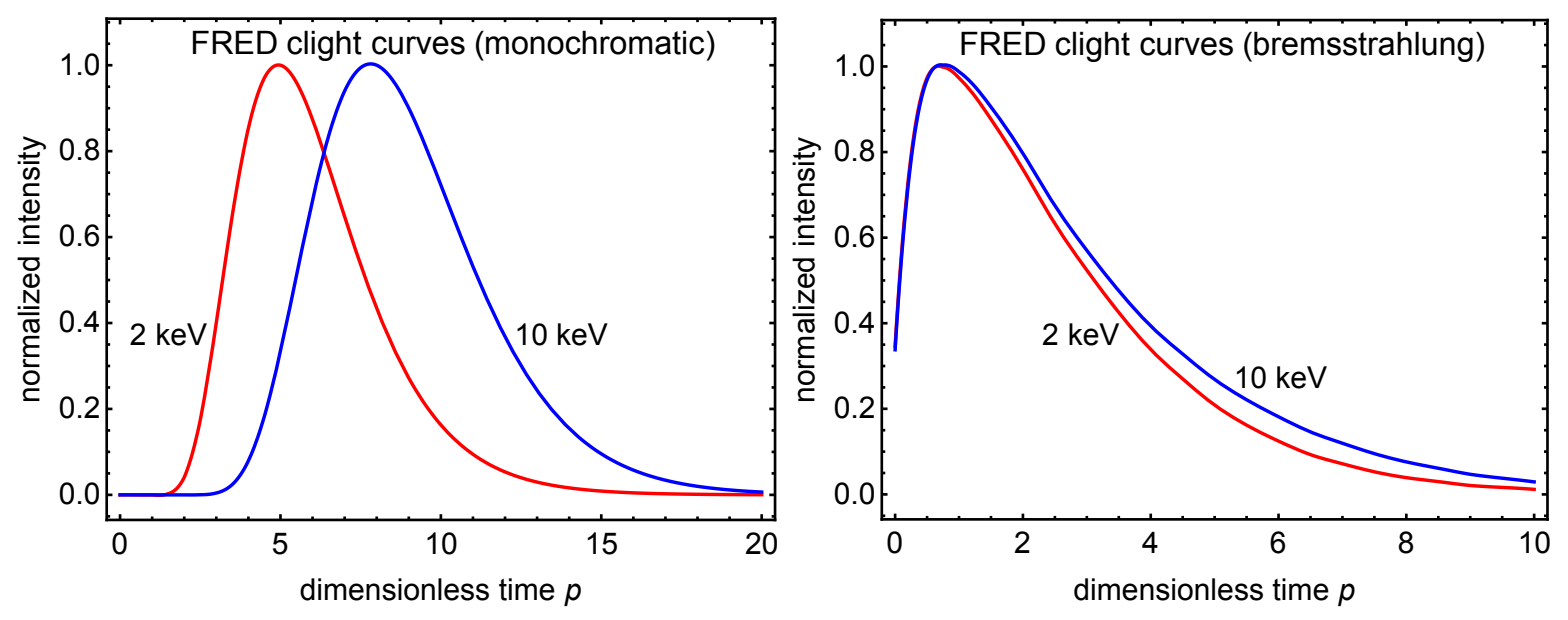

Fig. 9.-: FRED curves from monochromatic and bremsstrahlung injection in a homogeneous cloud. In each case, the red curve represents the soft energy channel, set at $2 \mathrm{keV}$, and the blue curve denotes the hard channel, set at $10 \mathrm{keV}$. The normalized intensity in each channel shows the relative lag.

The relationship between the two FRED light curves plotted in Figure 9 for the case of bremsstrahlung injection is qualitatively different from the monochromatic example. In this case, the initial fast rise in both channels is coherent, meaning that the hard and soft channel signals track each other relatively closely. This results in a small time lag at high Fourier frequencies, because the fast rise portion of each curve represents the most rapid variation in the system. Physically, this part of the process corresponds to the prompt escape of "pristine" bremsstrahlung seed photons that are almost unaffected by scattering. Because bremsstrahlung is a broadband emission mechanism, both hard and soft photons exist in the initial distribution, and the prompt escape is therefore coherent across the energy channels. This is, of course, not true in the case of low-energy monochromatic injection, because in that scenario, photons require sufficient time to upscatter into both the soft and hard energy channels. 
At longer timescales (smaller Fourier frequencies) in the bremsstrahlung case, the hard light curve approaches a delayed version of the soft light curve, reflecting the time it takes for the photons to Compton upscatter to the hard channel energy. This part of the process is similar to the monochromatic case, and indeed, we see that the time lags level off to a plateau at small Fourier frequencies, just as in the monochromatic example. To summarize, the overall behavior of the bremsstrahlung injection model matches the observational data much more closely then does the monochromatic injection scenario because of the combination of prompt escape (the fast rise part of the light curves) along with Compton reverberation (exponential decay) on longer timescales. This explains the origin of the qualitative difference in the behavior of the time lags at high Fourier frequencies exhibited in the monochromatic and bremsstrahlung injection scenarios, depicted in Figures 5 and 6, respectively.

\subsection{Coronal Temperature}

Both our model and that analyzed by HKC require the presence of hot electrons with temperature $T_{e} \sim 10^{8} \mathrm{~K}$ at distances $r \sim 10^{3} \mathrm{GM} / \mathrm{c}^{2}$ from the black hole. This temperature distribution is consistent with a substantial number of studies that focus on energy transport in inefficient accretion flows, with accretion rates that are significantly sub-Eddington, as first established by Nayaran \& Yi (1995) in the context of the original, self-similar Advection Dominated Accretion Flow (ADAF) model. Similar results for the temperature distribution were later obtained using more complex numerical simulations by Oda et al. (2012), Rajesh \& Mukhopadhyay (2010), Yuan et al. (2006), Mandal \& Chakrabarti (2005), Liu et al. (2002), Różańska \& Czerny (2000), and You et al. (2012). In these hot ADAF flows, the density in the outer region is so low that bremsstrahlung and inverse-Compton cooling are very inefficient. The lack of efficient cooling drives the 
electron temperature in the corona close to the virial value, out to distances of hundreds or thousands of gravitational radii from the black hole, in agreement with the temperature profiles assumed here.

In the study presented here, we have assumed that the electron scattering corona is isothermal in order to accomplish the separation of variables that is required to obtain analytical solutions to the radiation transport equation. The resulting analytical solutions allow us to determine the physical properties of the scattering corona in a given source by computing the time-averaged X-ray spectrum and the time-lag profile and comparing the theoretical results with the observational data. The assumption of an isothermal corona is roughly justified by studies indicating that the temperature does not vary by more than a factor of a few across the corona (e.g., You et al. 2012; Schnittman et al. 2013). Nonetheless, it is worth asking whether our results would be significantly modified in the presence of a coronal temperature gradient.

If the electron temperature varied across the corona, then in general one would expect the plasma to be hotter in the inner region, where the density is likely to be higher as well. In this scenario, the photons in the hot inner region would Compton upscatter faster than those in the cooler outer region, but they would spend more time (on average) scattering through the cloud before escaping due to the greater optical depth in the inner region. We estimate that these two effects would roughly offset each other, leaving the time lag profile close to the isothermal result derived here, if the temperature were set equal to the average value in the corona. Hence we predict that the results obtained for the time lags in the presence of a temperature gradient would be qualitatively similar to those obtained here using the isothermal assumption. Moreover, while the electrons may approach the virial temperature in the outer region, it is likely that in the inner region, the electron temperature is thermostatically controlled by Compton scattering (e.g., Sunyaev \& 
Titarchuk 1980; Shapiro, Lightman \& Eardley 1976). The combination of these two effects will tend to produce a relatively high, but uniform, electron temperature distribution, as we have assumed here.

\subsection{Time Varying Coronal Parameters}

If the transients responsible for producing the observed X-ray time lags in accreting black hole sources are driven by the deposition of a large amount of energy, then the properties of the corona (temperature, density) would be expected to respond. If this response occurs on time scales comparable to the diffusion time for photons to escape from the corona, then the resulting time lag profiles would be modified compared with the results obtained here, since we assume that the properties of the corona remain constant. Malzac \& Jourdain (2000) have considered the possible variation of the coronal properties during X-ray flares using a non-linear Monte Carlo simulation to study the flare evolution as a function of time, along with the associated variation of the temperature and optical depth in the corona. They do not compute Fourier time lags, but they do present simulated light curves in the soft and hard energy channels. In their model, the flares are driven by a sudden increase in the disk's internal dissipation, which produces a large quantity of soft photons. The temperature and optical depth of the corona change self-consistently during the transient, and then return to the equilibrium state. They find that hard time lags are produced during the flare if the energy deposition is substantial.

The approach taken by Malzac \& Jourdain (2000) is based on the pulse-avalanche model of Poutanen \& Fabian (1999). The model does not explicitly include Compton upscattering as a contributor to the time-lag phenomenon, nor was the significance of the injection spectrum considered. The simulated light curves generated by Malzac \& Jourdain (2000) sometimes display temporal dips, but the time dependence doesn't seem to resemble 
that observed during the transients in Cyg X-1. Since these authors do not compute Fourier time lags, it is difficult to directly compare their results with ours. However, we note that the transients under study here represent relatively small variations in the X-ray luminosity, which suggests that the energy deposition may not be large enough to significantly alter the large-scale properties of the scattering corona during the time it takes the photons to diffuse out of the cloud (Nowak et a. 1999; Cassatella et al. 2012). This supports our assumption that the temperature and density of the corona remain essentially constant during the formation of the observed time lags.

\subsection{Conclusion}

Our goal in this paper is to develop an integrated model, based on the diffusion and thermal Comptonization of seed photons in an optically-thick scattering cloud, that can naturally reproduce both the observed X-ray spectra and the time lags for Cyg X-1 and GX 339-04 using a single set of cloud parameters (density, radius, temperature). We have derived and presented a new set of exact mathematical solutions describing the Comptonization of seed photons injected into a scattering cloud of finite size that is either homogeneous, or possesses an electron number density that varies with radius as

$n_{e}(r) \propto r^{-1}$. The results developed here include new expressions for (a) the Green's function describing the radiated time-averaged X-ray flux (corresponding to the reprocessing of continually injected monochromatic seed photons), (b) the Green's function for the Fourier transform of the time-dependent radiation spectrum resulting from the impulsive injection of monochromatic seed photons, and (c) the associated X-ray Fourier time lags.

By exploiting the linearity of the fundamental transport equation, we used our results for the Green's function to explore a variety of seed photon injection scenarios. One of our main conclusions is that the integrated model can successfully explain the data regardless 
of the cloud configuration (homogeneous or inhomogeneous), provided the optical thickness and the temperature are comparable in the two models, as expected based on the Compton reverberation scenario (Payne 1980). Our results demonstrate that the bremsstrahlung injection model fits the observational time-lag data reasonably well for both Cyg X-1 and GX 339-04, whether the scattering corona is homogeneous or inhomogeneous. We therefore conclude that the constant time lags found by HKC in the homogeneous cloud configuration were the result of their utilization of a quasi-monochromatic (low-temperature blackbody) injection spectrum for the seed photon distribution.

The injection location in our model is different from that considerd by HKC, who assumed that the seed photons were always injected at the center of the spherical cloud. In our model, the injection location is arbitrary, and we find that the best agreement with the time lag data is obtained when the injection is relatively close to the surface of the cloud, so that the prompt escape of some of the unprocessed bremsstrahlung seed photons is able to explain the diminishing time lags observed at high Fourier frequencies. At longer timescales, the standard thermal Comptonization process sets the delay between the soft and hard channels, and this naturally leads to the observed plateau in the time lags at low Fourier frequencies.

In future work, we plan to develop a more general Green's function in which the injection occurs on a ring or a point, rather than on a spherical shell as in the model considered here. As in the present paper, the resulting Fourier transform of the timedependent Green's function in the general case will allow us to investigate a variety of seed photon energy distributions (e.g., blackbody or bremsstrahlung). The additional geometric flexibility in the general model should allow us to further improve the agreement between the model predictions and the data, hence providing new insights into the structure of the scattering corona and the underlying accretion disk. We also plan to examine scenarios in 
which the electrons cool during the transient in response to the upscattering of the injected photons. This may help to explain the soft time lags observed in some accreting black-hole sources (e.g., Fabian et al. 2009).

The authors are grateful to the anonymous referee who provided a variety of insightful comments that helped to strengthen and clarify the results presented here.

\section{Appendix A}

In order to use the series expansions developed in Sections 3 and 4 to represent the Green's functions for the time-averaged (quiescent) spectrum and for the Fourier transform of the time-dependent spectrum, it is necessary to establish the orthogonality of the various spatial eigenfunctions. In this section, we present a global proof of orthogonality of the spatial eigenfunctions for both the homogeneous case (utilizing the mirror inner boundary condition) and for the inhomogeneous case (utilizing the dual free-streaming boundary condition). First we define the generic spatial ODE, encompassing Equations (46)), (69), (87), and (106), by writing

$$
\frac{1}{z^{2-\alpha}} \frac{d}{d z}\left(z^{2+\alpha} \frac{d \Gamma_{n}}{d z}\right)+\eta^{2} \xi_{n} \Gamma_{n}(z)=0,
$$

such that,

$$
\begin{gathered}
\alpha= \begin{cases}0, & \text { homogeneous (quiescent \& Fourier transform) }, \\
1, & \text { inhomogeneous (quiescent \& Fourier transform) },\end{cases} \\
\Gamma_{n}(z)= \begin{cases}Y_{n}(z), & \text { homogeneous (quiescent \& Fourier transform) } \\
y_{n}(z), & \text { inhomogeneous (quiescent) } \\
g_{n}(z), & \text { inhomogeneous (Fourier transform) },\end{cases}
\end{gathered}
$$


and

$$
\xi_{n}= \begin{cases}\lambda_{n}, & \text { homogeneous (quiescent \& Fourier transform) } \\ \lambda_{n}, & \text { inhomogeneous (quiescent) } \\ \lambda_{n}+3 i \tilde{\omega} z, & \text { inhomogeneous (Fourier transform) } .\end{cases}
$$

To establish orthogonality, we multiply Equation (154) by $\Gamma_{m}(z)$ and then duplicate it with the indices exchanged, after which we subtract the second equation from the first, yielding

$$
\Gamma_{m} \frac{d}{d z}\left(z^{2+\alpha} \frac{d \Gamma_{n}}{d z}\right)-\Gamma_{n} \frac{d}{d z}\left(z^{2+\alpha} \frac{d \Gamma_{m}}{d z}\right)=-\eta^{2} z^{2-\alpha}\left(\xi_{n}-\xi_{m}\right) \Gamma_{n}(z) \Gamma_{m}(z) .
$$

Next, we integrate by parts with respect to $z$ over the computational domain $z_{\text {in }} \leq z \leq 1$ to obtain, after simplification,

$$
\left.\left(z^{2+\alpha} \Gamma_{m} \frac{d \Gamma_{n}}{d z}-z^{2+\alpha} \Gamma_{n} \frac{d \Gamma_{m}}{d z}\right)\right|_{z_{\mathrm{in}}} ^{1}=-\eta^{2}\left(\xi_{n}-\xi_{m}\right) \int_{z_{\mathrm{in}}}^{1} z^{2-\alpha} \Gamma_{n}(z) \Gamma_{m}(z) d z .
$$

The left-hand side of Equation (159) needs to be evaluated separately for the homogeneous and inhomogeneous cases, since the spatial boundary conditions are different in the two situations. We consider each of these cases in turn below.

For the homogeneous cloud configuration, with $\alpha=0$ and $z_{\text {in }}=0$, the inner and outer spatial boundary conditions can be written as (cf. Equations (51) and (53))

$$
\lim _{z \rightarrow 0} z^{2} \frac{d \Gamma_{n}}{d z}=0, \quad \lim _{z \rightarrow 1}\left[\frac{1}{3 \eta} \frac{d \Gamma_{n}}{d z}+\Gamma_{n}\right]=0
$$

Likewise, in the inhomogeneous case, with $\alpha=1$, we can express the inner and outer boundary conditions as (cf. Equations (92) and (94))

$$
\lim _{z \rightarrow z_{\text {in }}}\left[\frac{z}{3 \eta} \frac{d \Gamma_{n}}{d z}-\Gamma_{n}\right]=0, \quad \lim _{z \rightarrow 1}\left[\frac{z}{3 \eta} \frac{d \Gamma_{n}}{d z}+\Gamma_{n}\right]=0
$$

Using either the homogeneous or inhomogeneous boundary conditions given by Equations (160) and (161), respectively, we find that the left-hand side of Equation (159) 
vanishes, which establishes the required orthogonality of the spatial eigenfunctions. The orthogonality condition can be written in general as

$$
\int_{z_{\mathrm{in}}}^{1} z^{2-\alpha} \Gamma_{n}(z) \Gamma_{m}(z) d z=0, \quad n \neq m
$$

\section{Appendix B}

As shown in Section 5, the particular solution for the Fourier transform in the case of bremsstrahlung, $F_{\text {brem }}$, injection is given by the convolution (see Equation (128)

$$
F_{\text {brem }}\left(x, z, z_{0}, \tilde{\omega}\right)=\int_{x_{\text {abs }}}^{\infty} F_{\mathrm{G}}\left(x, x_{0}, z, z_{0}, \tilde{\omega}\right) A_{0} x_{0}^{-1} e^{-x_{0}} N_{0}^{-1} d x_{0},
$$

where $x_{\text {abs }}$ is the dimensionless self-absorption cutoff energy, the constant $A_{0}$ is given by Equation (127), and the Fourier transform Green's function, $F_{\mathrm{G}}$, is given by Equations (833) and (123) in the homogeneous and inhomogeneous cases, respectively. In general, we can write $F_{\mathrm{G}}$ in the generic form

$$
F_{\mathrm{G}}\left(x, x_{0}, z, z_{0}, \tilde{\omega}\right)=N_{0} e^{\left(x_{0}-x\right) / 2}\left(x x_{0}\right)^{-2} \sum_{n=0}^{\infty} M_{2, \lambda}\left(x_{\min }\right) W_{2, \lambda}\left(x_{\max }\right) \mathscr{A}_{n}\left(z, z_{0}, \tilde{\omega}\right),
$$

where $x_{\min }=\min \left(x, x_{0}\right), x_{\max }=\max \left(x, x_{0}\right)$, and $\mathscr{A}_{n}$ is a composite function containing the expansion coefficients and the spatial eigenfunctions, given by

$$
\mathscr{A}_{n}\left(z, z_{0}, \tilde{\omega}\right)=\frac{e^{i \tilde{\omega} p_{0}} \eta^{3}}{4 \pi R^{3} \Theta^{4}\left(m_{e} c^{2}\right)^{3}} \begin{cases}\frac{\Gamma(\mu-3 / 2) Y_{n}\left(z_{0}\right) Y_{n}(z)}{\Gamma(1+2 \mu) \mathscr{I}_{n}}, & \text { homogeneous }, \\ \frac{\Gamma(\sigma-3 / 2) g_{n}\left(z_{0}\right) g_{n}(z)}{\Gamma(1+2 \sigma) \eta^{3} \mathscr{K}_{n}}, & \text { inhomogeneous } .\end{cases}
$$

In the homogeneous case, $\mu$ is computed using Equation (76), and in the inhomogeneous case, $\sigma$ is computed using Equation (109). Combining Equations (163) and (164), and reversing the order of summation and integration, we obtain

$$
F_{\mathrm{brem}}\left(x, z, z_{0}, \tilde{\omega}\right)=A_{0} e^{-x / 2} x^{-2} \sum_{n=0}^{\infty} \mathscr{A}_{n}\left(z, z_{0}, \tilde{\omega}\right) B(\lambda, x),
$$


where

$$
B(\lambda, x) \equiv \int_{x_{\text {abs }}}^{\infty} e^{-x_{0} / 2} x_{0}^{-3} M_{2, \lambda}\left(x_{\min }\right) W_{2, \lambda}\left(x_{\max }\right) d x_{0}
$$

and we set $\lambda=\mu$ to treat the homogeneous case, and we set $\lambda=\sigma$ to treat the inhomogeneous case.

Our remaining task is to evaluate the integral function $B$ analytically, if possible. The expression for $B$ can be broken into two integrals by writing, for $x \geq x_{\mathrm{abs}}$,

$$
B(\lambda, x)=\left.I_{M}\left(\lambda, x_{0}\right)\right|_{x_{\mathrm{abs}}} ^{x} W_{2, \lambda}(x)+\left.I_{W}\left(\lambda, x_{0}\right)\right|_{x} ^{\infty} M_{2, \lambda}(x)
$$

and, for $x \leq x_{\mathrm{abs}}$,

$$
B(\lambda, x)=\left.I_{W}\left(\lambda, x_{0}\right)\right|_{x_{\mathrm{abs}}} ^{\infty} M_{2, \lambda}(x)
$$

where we have defined the indefinite integrals $I_{M}\left(\lambda, x_{0}\right)$ and $I_{W}\left(\lambda, x_{0}\right)$ using

$$
I_{M}\left(\lambda, x_{0}\right) \equiv \int e^{-x_{0} / 2} x_{0}^{-3} M_{2, \lambda}\left(x_{0}\right) d x_{0}, \quad I_{W}\left(\lambda, x_{0}\right) \equiv \int e^{-x_{0} / 2} x_{0}^{-3} W_{2, \lambda}\left(x_{0}\right) d x_{0} .
$$

It is convenient to rewrite the Whittaker functions in the integrands for $I_{M}$ and $I_{W}$ using the Kummer function identities (Abramowitz \& Stegun 1970),

$$
\begin{aligned}
& M_{\alpha, \beta}(z)=e^{-z / 2} z^{\frac{1}{2}+\beta} M\left(\frac{1}{2}+\beta-\alpha, 1+2 \beta, z\right) \\
& W_{\alpha, \beta}(z)=e^{-z / 2} z^{\frac{1}{2}+\beta} U\left(\frac{1}{2}+\beta-\alpha, 1+2 \beta, z\right)
\end{aligned}
$$

which yield

$$
I_{M}(\lambda, x)=\int e^{-x} x^{b-a-5} M(a, b, x) d x, \quad I_{W}(\lambda, x)=\int e^{-x} x^{b-a-5} U(a, b, x) d x,
$$

where

$$
a=\lambda-\frac{3}{2}, \quad b=2 \lambda+1
$$

The integral $I_{W}(\lambda, x)$ can be carried out analytically using Slater's (1960) identity,

$$
\int e^{-x} x^{b-a-2} U(a, b, x) d x=-e^{-x} x^{b-a-1} U(a+1, b, x) .
$$


Integrating Equation (173) by parts once yields

$$
\int x^{-3} e^{-x} x^{b-a-2} U(a, b, x) d x=-x^{-3} e^{-x} x^{b-a-1} U(a+1, b, x)-3 \int x^{-4} e^{-x} x^{b-a-1} U(a+1, b, x) d x .
$$

Integrating by parts again gives

$$
\begin{array}{r}
\int x^{-3} e^{-x} x^{b-a-2} U(a, b, x) d x=-x^{-3} e^{-x} x^{b-a-1} U(a+1, b, x)-3\left[-x^{-2} e^{-x} x^{b-a^{\prime}-1} U\left(a^{\prime}+1, b, x\right)\right. \\
\left.-2 \int x^{-3} e^{-x} x^{b-a^{\prime}-1} U\left(a^{\prime}+1, b, x\right) d x\right], \quad(177)
\end{array}
$$

where $a^{\prime}=a+1$. Integrating by parts a third time yields

$$
\begin{gathered}
\int x^{-3} e^{-x} x^{b-a-2} U(a, b, x) d x=-x^{-3} e^{-x} x^{b-a-1} U(a+1, b, x)-3\left\{-x^{-2} e^{-x} x^{b-a^{\prime}-1} U\left(a^{\prime}+1, b, x\right)\right. \\
\left.-2\left[-x^{-1} e^{-x} x^{b-a^{\prime \prime}-1} U\left(a^{\prime \prime}+1, b, x\right)-\int x^{-2} e^{-x} x^{b-a^{\prime \prime}-1} U\left(a^{\prime \prime}+1, b, x\right) d x\right]\right\}
\end{gathered}
$$

where $a^{\prime \prime}=a^{\prime}+1$. The remaining integral can be evaluated directly using Equation (175) to obtain, after some algebra,

$$
\begin{aligned}
\int x^{-3} e^{-x} x^{b-a-2} U(a, b, x) d x=e^{-x} x^{b-a-4}[-U(a+1, b, x)+3 U(a+2, b, x) \\
-6 U(a+3, b, x)+6 U(a+4, b, x)] .
\end{aligned}
$$

By converting the Kummer functions to Whittaker functions, we obtain the final expression

$$
I_{W}(\lambda, x)=e^{-x / 2} x^{-2}\left[-W_{1, \lambda}(x)+3 W_{0, \lambda}(x)-6 W_{-1, \lambda}(x)+6 W_{-2, \lambda}(x)\right] .
$$

Likewise, the integral $I_{M}(\lambda, x)$ in Equation (173) can be evaluated using Slater's (1960) identity

$$
\int e^{-x} x^{b-a-2} M(a, b, x) d x=\frac{e^{-x} x^{b-a-1}}{b-a-1} M(a+1, b, x) .
$$

Following the same iterative procedure used to evaluate $I_{W}(\lambda, x)$, we eventually arrive at 
the result

$$
\begin{aligned}
I_{M}(\lambda, x)=\frac{e^{-x} x^{b-a-4}}{b-a-1}( & M(a+1, b, x)+\frac{3}{b-a-2}\{M(a+2, b, x) \\
& \left.\left.+\frac{2}{b-a-3}\left[M(a+3, b, x)+\frac{1}{b-a-4} M(a+4, b, x)\right]\right\}\right),
\end{aligned}
$$

which can be rewritten in terms of the Whittaker functions as

$$
I_{M}(\lambda, x)=\frac{x^{-2} e^{-x / 2}}{\lambda+\frac{3}{2}}\left(M_{1, \lambda}(x)+\frac{3}{\lambda+\frac{1}{2}}\left\{M_{0, \lambda}(x)+\frac{2}{\lambda-\frac{1}{2}}\left[M_{-1, \lambda}(x)+\frac{1}{\lambda-\frac{3}{2}} M_{-2, \lambda}(x)\right]\right\}\right) .
$$

Our final expression for the integral function $B(\lambda, x)$ is obtained by rewriting Equations (168) and (169) as

$$
B(\lambda, x)= \begin{cases}W_{2, \lambda}(x)\left[I_{M}(\lambda, x)-I_{M}\left(\lambda, x_{\mathrm{abs}}\right)\right]-M_{2, \lambda}(x) I_{W}(\lambda, x), & x \geq x_{\mathrm{abs}} \\ -M_{2, \lambda}(x) I_{W}\left(\lambda, x_{\mathrm{abs}}\right), & x \leq x_{\mathrm{abs}}\end{cases}
$$

where $I_{W}(\lambda, x)$ and $I_{M}(\lambda, x)$ are evaluated using Equations (180) and (183), respectively. We can now combine Equations (165) and (166) to express the bremsstrahlung injection Fourier transform $F_{\text {brem }}$ as $F_{\text {brem }}\left(x, z, z_{0}, \tilde{\omega}\right)=\frac{e^{i \tilde{\omega} p_{0}} \eta^{3} A_{0} e^{-x / 2}}{4 \pi R^{3} \Theta^{4}\left(m_{e} c^{2}\right)^{3} x^{2}} \sum_{n=0}^{\infty} \begin{cases}\frac{\Gamma(\mu-3 / 2) Y_{n}\left(z_{0}\right) Y_{n}(z)}{\Gamma(1+2 \mu) \mathscr{I}_{n}} B(\mu, x), & \text { homogeneous }, \\ \frac{\Gamma(\sigma-3 / 2) g_{n}\left(z_{0}\right) g_{n}(z)}{\Gamma(1+2 \sigma) \eta^{3} \mathscr{K}_{n}} B(\sigma, x), & \text { inhomogeneous }\end{cases}$

where $B(\mu, x)$ and $B(\sigma, x)$ are evaluated using Equation (184). 


\section{REFERENCES}

Abramowitz, M., \& Stegun, I. A. 1970, Handbook of Mathematical Functions, (New York: Dover)

Becker, P. A., 2003, MNRAS, 343, 215

Cadolle Bel, M., Rodriguez, J., D’Avanzo, P., et al. 2011, A\&A, 534, 119

Cadolle Bel, M., Sizun, P., Goldwurm, A., et al. 2006, A\&A, 446, 591

Cassatella. P., Uttley, P., Wilms, J., \& Poutanen, J. 2012, MNRAS, 422, 2407

Del Santo, M., Malzac, J., et al. 2013, MNRAS, 430, 209

Fabian, A. C., Zoghbi, A., Ross, R. R., et al. 2009, Nature, 459, 540

Frontera, F., Palazzi, E., Zdziarski, A. A. et al. 2001, ApJ, 546, 1027

Ter Haar, D., \& Wergeland, H. 1971, PhysRep, 1, 31

Hua, X.-M. 1997, CompPh, 11, 660

Hua, X.-M., Kazanas, D., \& Cui, W. 1999, ApJ, 512, 793

Kroon, J. J., \& Becker, P. A. 2014, ApJL, 785, L34

Ling, J. C., Wheaton, W. A., Wallyn, P., et al. 1997, ApJ, 484, 375

Liu, B. F., Mineshige, S., et al. 2002, ApJ, 575, 117

Malzac, J. 2012, IJMP, 8, 73

Malzac, J., \& Jourdain, E. 2000, A\&A, 359, 843

Malzac, J., Lubiński, P., et al. 2008, A\&A, 492, 527 
Mandal, S., \& Chakrabarti, S. K. 2005, A\&A, 434, 839

Meyer, F., Liu, B. F., \& Meyer-Hofmeister, E. 2007, A\&A, 463, 1

Miyamoto, S., Kitamoto, S., Mitsuda, K., \& Dotani, T. 1988, Nature, 336, 450

Nayaran, R., \& Yi, I. 1995, ApJ, 452, 710

Nowak, M. A., Vaughan, B. A., Wilms, J., Dove, J. B., \& Begelman, M. C. 1999, ApJ, 510, 874

Oda, H., Machida, M., et al. 2012, PASJ, 64, 15

Payne, D.G. 1980, ApJ, 237, 951

Poutanen, J., \& Fabian, A. C. 1999, MNRAS, 306, L31

Rajesh, S. R., \& Mukhopadhyay, B. 2010, MNRAS, 402, 961

Różańska, A., \& Czerny, B. 2000, A\&A, 360, 1170

Rybicki, G. B., \& Lightman, A. P. 1979, Radiative Processes in Astrophysics (New York: Wiley)

Schnittman, J. D., Krolik, J. H., \& Noble, S. C. 2013, ApJ, 769, 156

Shapiro, S. L., Lightman, A. P., \& Eardley 1976, ApJ, 204, 187

Slater L. J. 1960, Confluent Hypergeometric Functions. (Cambridge: Cambridge Univ. Press)

Sunyaev, R. A., \& Titarchuk, L. G. 1980, A\&A, 86, 121

van der Klis, M., Hasinger, G., Stella, L., et al. 1987, ApJL, 319, L13

You, B., Cao, X., \& Yuan, Y.-F. 2012, ApJL, 761, 109 
Yuan, F., Taam, R. E., et al. 2006, ApJ, 636, 46 